\title{
"REFSDAL" MEETS POPPER: COMPARING PREDICTIONS OF THE RE-APPEARANCE OF THE MULTIPLY IMAGED SUPERNOVA BEHIND MACSJ1149.5+2223
}

\author{
T. Treu ${ }^{1,28}$, G. Brammer ${ }^{2}$, J. M. Diego ${ }^{3}$, C. Grillo ${ }^{4}$, P. L. Kelly ${ }^{5}$, M. Oguri ${ }^{6,7,8}$, S. A. Rodney ${ }^{9,10,29}$, P. Rosati ${ }^{11}$, \\ K. Sharon ${ }^{12}$, A. Zitrin ${ }^{13,29}$, I. Balestra ${ }^{14}$, M. Bradač ${ }^{15}$, T. Broadhurst ${ }^{16,17}$, G. B. Caminha ${ }^{11}$, A. Halkola, A. Hoag ${ }^{15}$,

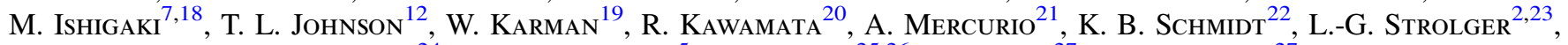 \\ S. H. Suyu ${ }^{24}$, A. V. Filippenko ${ }^{5}$, R. J. Foley ${ }^{25,26}$, S. W. Jha ${ }^{27}$, And B. Patel ${ }^{27}$ \\ ${ }^{1}$ Department of Physics and Astronomy, University of California, Los Angeles, CA 90095, USA; tt@astro.ucla.edu \\ ${ }^{2}$ Space Telescope Science Institute, 3700 San Martin Drive, Baltimore, MD 21218, USA \\ ${ }^{3}$ IFCA, Instituto de Física de Cantabria (UC-CSIC), Av. de Los Castros s/n, E-39005 Santander, Spain \\ ${ }^{4}$ Dark Cosmology Centre, Niels Bohr Institute, University of Copenhagen, Juliane Maries Vej 30, DK-2100 Copenhagen, Denmark \\ ${ }^{5}$ Department of Astronomy, University of California, Berkeley, CA 94720-3411, USA \\ ${ }^{6}$ Kavli Institute for the Physics and Mathematics of the universe (Kavli IPMU, WPI), University of Tokyo, 5-1-5 Kashiwanoha, Kashiwa, Chiba 277-8583, Japan \\ 7 Department of Physics, University of Tokyo, 7-3-1 Hongo, Bunkyo-ku, Tokyo 113-0033, Japan \\ ${ }^{8}$ Research Center for the Early universe, University of Tokyo, 7-3-1 Hongo, Bunkyo-ku, Tokyo 113-0033, Japan \\ ${ }^{9}$ Department of Physics and Astronomy, University of South Carolina, 712 Main Street, Columbia, SC 29208, USA \\ ${ }^{10}$ Department of Physics and Astronomy, The Johns Hopkins University, 3400 N. Charles Street, Baltimore, MD 21218, USA \\ ${ }^{11}$ Dipartimento di Fisica e Scienze della Terra, Università degli Studi di Ferrara, via Saragat 1, I-44122, Ferrara, Italy \\ ${ }^{12}$ Department of Astronomy, University of Michigan, 1085 S. University Avenue, Ann Arbor, MI 48109, USA \\ ${ }^{13}$ California Institute of Technology, 1200 East California Boulevard, Pasadena, CA 91125, USA \\ ${ }^{14}$ University Observatory Munich, Scheinerstrasse 1, D-81679 Munich, Germany \\ ${ }^{15}$ University of California Davis, 1 Shields Avenue, Davis, CA 95616, USA \\ ${ }^{16}$ Fisika Teorikoa, Zientzia eta Teknologia Fakultatea, Euskal Herriko Unibertsitatea Upv/Ehu, Spain \\ ${ }_{17}^{17}$ IKERBASQUE, Basque Foundation for Science, Alameda Urquijo, 36-5 E-48008 Bilbao, Spain \\ 18 Institute for Cosmic Ray Research, The University of Tokyo, Kashiwa, Chiba 277-8582, Japan
${ }^{19}$ Kapteyn Astronomical Institute, University of Groningen, Postbus 800, 9700 AV Groningen, The Netherlands \\ ${ }^{20}$ Department of Astronomy, Graduate School of Science, The University of Tokyo, 7-3-1 Hongo, Bunkyo-ku, Tokyo 113-0033, Japan \\ 21 INAF, Osservatorio Astronomico di Bologna, via Ranzani1, I-40127 Bologna, Italy \\ ${ }^{22}$ Department of Physics, University of California, Santa Barbara, CA 93106-9530, USA \\ ${ }^{23}$ Department of Physics, Western Kentucky University, Bowling Green, KY 42101, USA \\ ${ }^{24}$ Institute of Astronomy and Astrophysics, Academia Sinica, P.O. Box 23-141, Taipei 10617, Taiwan \\ ${ }_{25}$ Astronomy Department, University of Illinois at Urbana- Champaign, 1002 W. Green Street, Urbana, IL 61801, USA \\ ${ }^{26}$ Department of Physics, University of Illinois at Urbana-Champaign, 1110 W. Green Street, Urbana, IL 61801, USA \\ ${ }^{27}$ Department of Physics and Astronomy, Rutgers, The State University of New Jersey, Piscataway, NJ 08854, USA \\ Received 2015 October 19; accepted 2015 November 24; published 2016 January 20
}

\begin{abstract}
Supernova "Refsdal," multiply imaged by cluster MACS1149.5+2223, represents a rare opportunity to make a true blind test of model predictions in extragalactic astronomy, on a timescale that is short compared to a human lifetime. In order to take advantage of this event, we produced seven gravitational lens models with five independent methods, based on Hubble Space Telescope (HST) Hubble Frontier Field images, along with extensive spectroscopic follow-up observations by HST, the Very Large and the Keck Telescopes. We compare the model predictions and show that they agree reasonably well with the measured time delays and magnification ratios between the known images, even though these quantities were not used as input. This agreement is encouraging, considering that the models only provide statistical uncertainties, and do not include additional sources of uncertainties such as structure along the line of sight, cosmology, and the mass sheet degeneracy. We then present the model predictions for the other appearances of supernova "Refsdal." A future image will reach its peak in the first half of 2016, while another image appeared between 1994 and 2004. The past image would have been too faint to be detected in existing archival images. The future image should be approximately one-third as bright as the brightest known image (i.e., $H_{\mathrm{AB}} \approx 25.7$ mag at peak and $H_{\mathrm{AB}} \approx 26.7$ mag six months before peak), and thus detectable in single-orbit $H S T$ images. We will find out soon whether our predictions are correct.
\end{abstract}

Key words: gravitational lensing: strong

Supporting material: machine-readable table

\section{INTRODUCTION}

In 1964 Sjur Resfdal speculated that a supernova (SN) multiply imaged by a foreground massive galaxy could be used to measure distances and, therefore, the Hubble constant (Refsdal 1964). The basic physics behind this phenomenon is very simple. According to Fermat's principle, in gravitational

\footnotetext{
28 Packard Fellow.

29 Pubble Fellow.
}

optics as in standard optics, multiple images form at the stationary points of the excess arrival time surface (Schneider 1985; Blandford \& Narayan 1986). The excess arrival time is the result of the competition between the geometric time delay and the Shapiro (1964) delay. The arrival time thus depends on the apparent position of the image in the sky as well as the gravitational potential. Since the arrival time is measured in seconds, while all the other lensing observables are measured in angles on the sky, their relationship depends on 
the angular diameter distance $D$. In the simplest case of singleplane lensing, the time delay between two images is proportional to the so-called time-delay distance, $D_{\mathrm{d}} D_{\mathrm{s}}\left(1+z_{\mathrm{d}}\right) / D_{\mathrm{ds}}$, where $\mathrm{d}$ and s represent the deflector and the source, respectively (see, e.g., for definitions Meylan et al. 2006; Treu 2010; Suyu et al. 2010).

Over the past decades, many authors have highlighted the importance and applications of identifying such events (e.g., Kolatt \& Bartelmann 1998; Holz 2001; Goobar et al. 2002; Bolton \& Burles 2003; Oguri \& Kawano 2003), computed rates and proposed search strategies (Linder et al. 1988; Sullivan et al. 2000; Oguri et al. 2003; Oguri \& Marshall 2010), and identified highly magnified SNe (Quimby et al. 2014).

Finally, 50 years after the initial proposal by Refsdal, the first multiply imaged SN was discovered in 2014 November (Kelly et al. 2015) in Hubble Space Telescope (HST) images of the cluster MACSJ1149.5+2223 (Ebeling et al. 2007; Smith et al. 2009; Zitrin \& Broadhurst 2009), taken as part of the Grism Lens Amplified Survey from Space (GLASS; GO13459, PI Treu; Schmidt et al. 2014; Treu et al. 2015), and aptly nicknamed "SN Refsdal." SN Refsdal was identified in difference imaging as four point sources that were not present in earlier images taken as part of the CLASH survey (Postman et al. 2012). Luckily, the event was discovered just before the beginning of an intensive imaging campaign as part of the Hubble Frontier Field (HFF) initiative (Coe et al. 2015; J. M. Lotz et al. 2015, in preparation). Additional epochs were obtained as part of the Frontier SN program (GO-13790, PI Rodney), and a Director Discretionary Time program (GO/ DD-14041, PI Kelly). The beautiful images that have emerged (Figure 1) are an apt celebration of the international year of light and the 100 year anniversary of the theory of general relativity (e.g., Treu \& Ellis 2015).

The gravitational lensing configuration of SN Refsdal is very remarkable. The SN exploded in one arm of an almost face-on spiral galaxy that is multiply imaged and highly magnified by the cluster gravitational potential $\left(z_{\mathrm{s}}=1489\right.$ and $z_{\mathrm{d}}=0542$; redshifts from Grillo et al. 2015a). Furthermore, the spiral arm hosting SN Refsdal happens to be sufficiently close to a cluster member galaxy that four additional multiple images are formed with average separation of the order of arcseconds, typical of galaxy-scale strong lensing. This set of four images close together in an "Einstein cross" configuration is where SN Refsdal has been detected so far (labeled S1-S4 in Figure 1). As we discuss below, the cluster-scale images are more separated in terms of their arrival time, with time delays that can be much longer than the duration of the event, and therefore it is consistent with the lensing interpretation that they have not yet been seen.

The original suggestion by Refsdal (1964) was to use such events to measure distances and therefore cosmological parameters, starting from the Hubble constant. While distances with interesting accuracy and precision have been obtained from gravitational time delays in galaxy-scale systems lensing quasars (e.g., Suyu et al. 2014), it is premature to attempt this in the case of SN Refsdal. The time delay is not yet known with precision comparable to that attained for lensed quasars (e.g., Tewes et al. 2013b), and the mass distribution of the cluster MACSJ1149.5+2223 is inherently much more complex than that of a single elliptical galaxy.

However, SN Refsdal gives us a unique opportunity to test the current mass models of MACSJ1149.5+2223, by conducting a textbook-like falsifiable experiment (Popper 2002). All of the models that have been published after the discovery of SN Refsdal (Diego et al. 2015; Jauzac et al. 2015; Kelly et al. 2015; Oguri 2015; Sharon \& Johnson 2015) predict that an additional image will form some time in the near future (close to image 1.2 of the host galaxy, shown in Figure 1). It could appear as early as 2015 October or in a few years. The field of MACSJ1149.5 +2223 is unobservable with HST at the time of submission of this paper, but observations will resume at the end of 2015 October as part of an approved Cycle 23 program (GO-14199, PI Kelly). We thus have the opportunity to carry out a true blind test of the models, if we act sufficiently fast. This test is similar in spirit to the test of magnification models using singly imaged SNe Ia (Nordin et al. 2014; Patel et al. 2014; Rodney et al. 2015). The uniqueness of our test lies in the fact that it is based on the prediction of an event that has not yet happened, and it is thus intrinsically blind and immune to experimenter bias.

The quality and quantity of data available to lens modelers have improved significantly since the discovery of SN Refsdal and the publication of the first modeling papers. As part of the HFF and follow-up programs, there are now significantly deeper HST images. Spectroscopy of hundreds of sources in the field (Figure 1) is available from HST grism data obtained as part of GLASS and SN Refsdal follow-up campaign (PI Kelly), from Multi-Unit Spectroscopic Explorer (MUSE) Very Large Telescope (VLT) Director's Discretionary Time follow-up observations (Prog. ID 294.A-5032, PI Grillo), and from follow-up observations with the DEep Imaging Multi-Object Spectrograph (DEIMOS Faber et al. 2003) on the Keck II Telescope (PI Jha).

The timing is thus perfect to ask the question: "Given stateof-the-art data and models, how accurately can we predict the arrival time and magnification of the next appearance of a multiply imaged SN?" Answering this question will give us an absolute measurement of the quality of present-day models, although one should keep in mind that this is a very specific test. The arrival time and especially the magnification of a point source depend strongly on the details of the gravitational potential in the vicinity of the images. Additional uncertainties in the time delay and magnification arise from the inhomogeneous distribution of mass along the line of sight (Suyu et al. 2010; Collett et al. 2013; Greene et al. 2013), the mass-sheet degeneracy and its generalizations (Falco et al. 1985; Schneider \& Sluse 2013, 2014; Suyu et al. 2014; Xu et al. 2015), and the residual uncertainties in cosmological parameters, especially the Hubble constant (Riess et al. 2011; Freedman et al. 2012). Average or global quantities of more general interest, such as the total volume behind the cluster, or the average magnification, are much less sensitive to the details of the potential around a specific point.

In order to answer this question in the very short amount of time available, the SN Refsdal follow-up team worked hard to reduce and analyze the new data. By 2015 May it was clear that the quality of the follow-up data would be sufficient to make substantial improvements to their lens models. Therefore, the follow-up team contacted the three other groups who had by then published predictions for SN Refsdal, and offered them the new data sets to update their models, as part of a concerted comparison effort. The five groups worked together to incorporate the new information into the lensing analysis, first by identifying and rigorously vetting new sets of multiple images, and then to promptly update their models to make a 


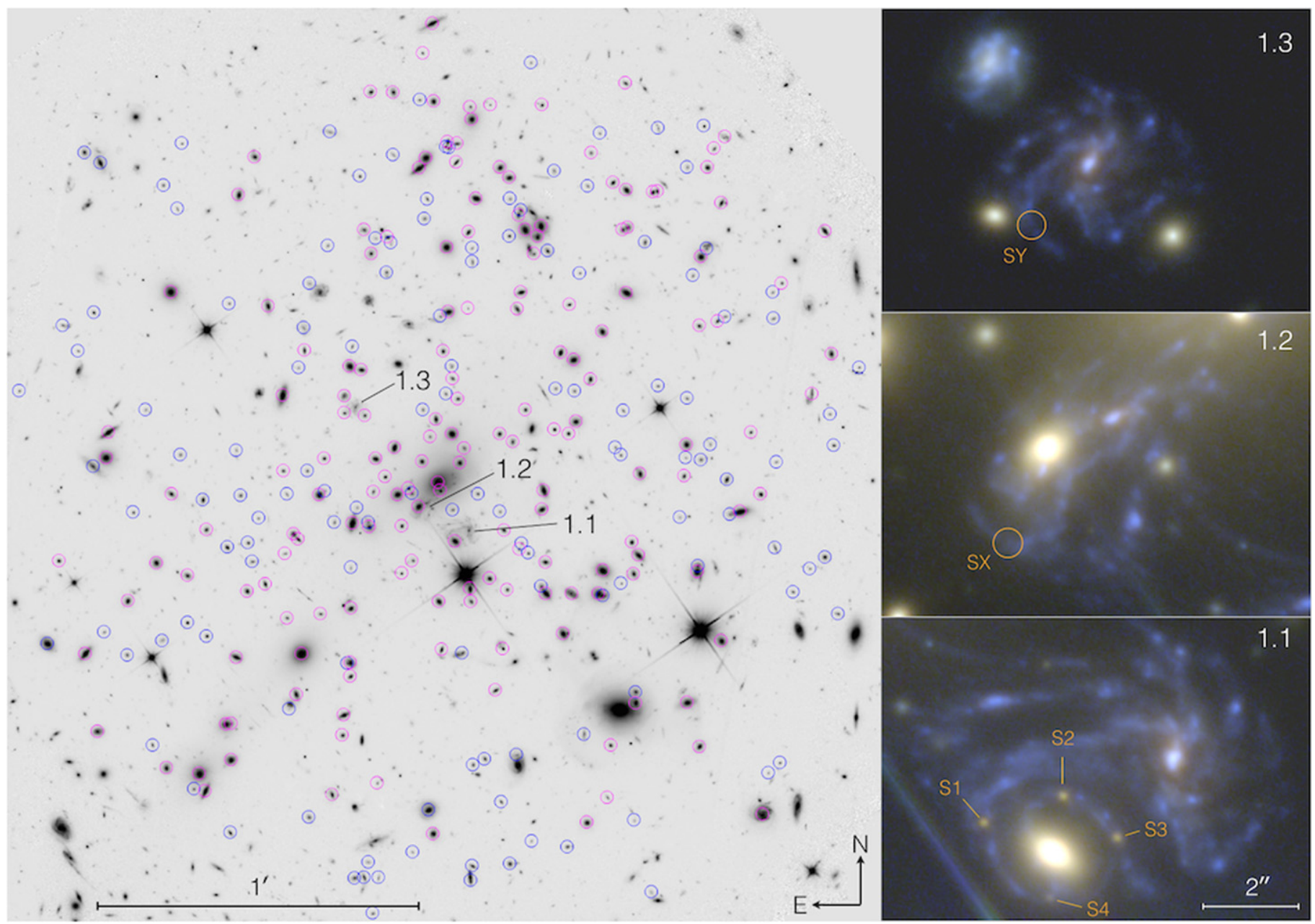

Figure 1. Multiple images of the SN Refsdal host galaxy behind MACSJ1149.5+2223. The left panel shows a wide view of the cluster, encompassing the entire footprint of the WFC3-IR camera. Spectroscopically confirmed cluster member galaxies are highlighted in magenta circles. Cyan circles indicate those associated with the cluster based on their photometric properties. The three panels on the right show in more detail the multiple images of the SN Refsdal host galaxy (labeled 1.1, 1.2, and 1.3). The positions of the known images of SN Refsdal are labeled as S1-S4, while the model-predicted locations of the future and past appearances are labeled as SX and SY, respectively.

timely prediction. A synopsis and comparison between the results and predictions of the various models is presented in this paper. Companion papers by the individual groups will describe the follow-up campaigns as well as the details of each modeling effort.

This paper is organized as follows. In Section 2, we briefly summarize the data sets and measurements that are used in this comparison effort. In Section 3, we review the constraints used by the modeling teams. Section 4 gives a concise description of each of the five lens modeling techniques adopted. Section 5 presents the main results of this paper-a comparison of the predictions of the different models. Section 6 discusses the results, and Section 7 concludes with a summary. To ensure uniformity with the modeling effort for the HFFs clusters, we adopt a concordance cosmology with $h=0.7, \quad \Omega_{m}=0.3, \quad$ and $\quad \Omega_{\Lambda}=0.7$. All magnitudes are given in the AB system (Oke \& Gunn 1983).

\section{SUMMARY OF DATA SETS AND MEASUREMENTS}

We summarize the data sets and measurements used in this paper. An overview of the field of view and pointing of the instruments used here is shown in Figure 2.

\subsection{HST Imaging}

Different versions of the images were used at different stages of the process. However, the final identifications of multiple images and their positions were based on the HFF data release v1.0, and their world coordinate system. The reader is referred to the HFF data release webpages ${ }^{30}$ for more information on these data.

\subsubsection{The Light Curves of SN Refsdal}

Since the discovery of SN Refsdal on 2014 November 11, the MACSJ1149.5+2223 field has been observed in great detail, with HST imaging in optical and infrared bands, and deep spectroscopy from HST, Keck, and the VLT. The main goal of the spectroscopic data is to determine the spectral classification of the SN (P. L. Kelly et al. 2015, in preparation). Photometry from the HST imaging provides well-sampled multi-color light curves for SN Refsdal images S1-S4 that exhibit a slow rise over $\sim 150$ days, reaching a peak brightness in the F160W band on approximately 2015 April 20, with an

\footnotetext{
${ }^{30} \mathrm{http}: / /$ www.stsci.edu/hst/campaigns/frontier-fields/
} 


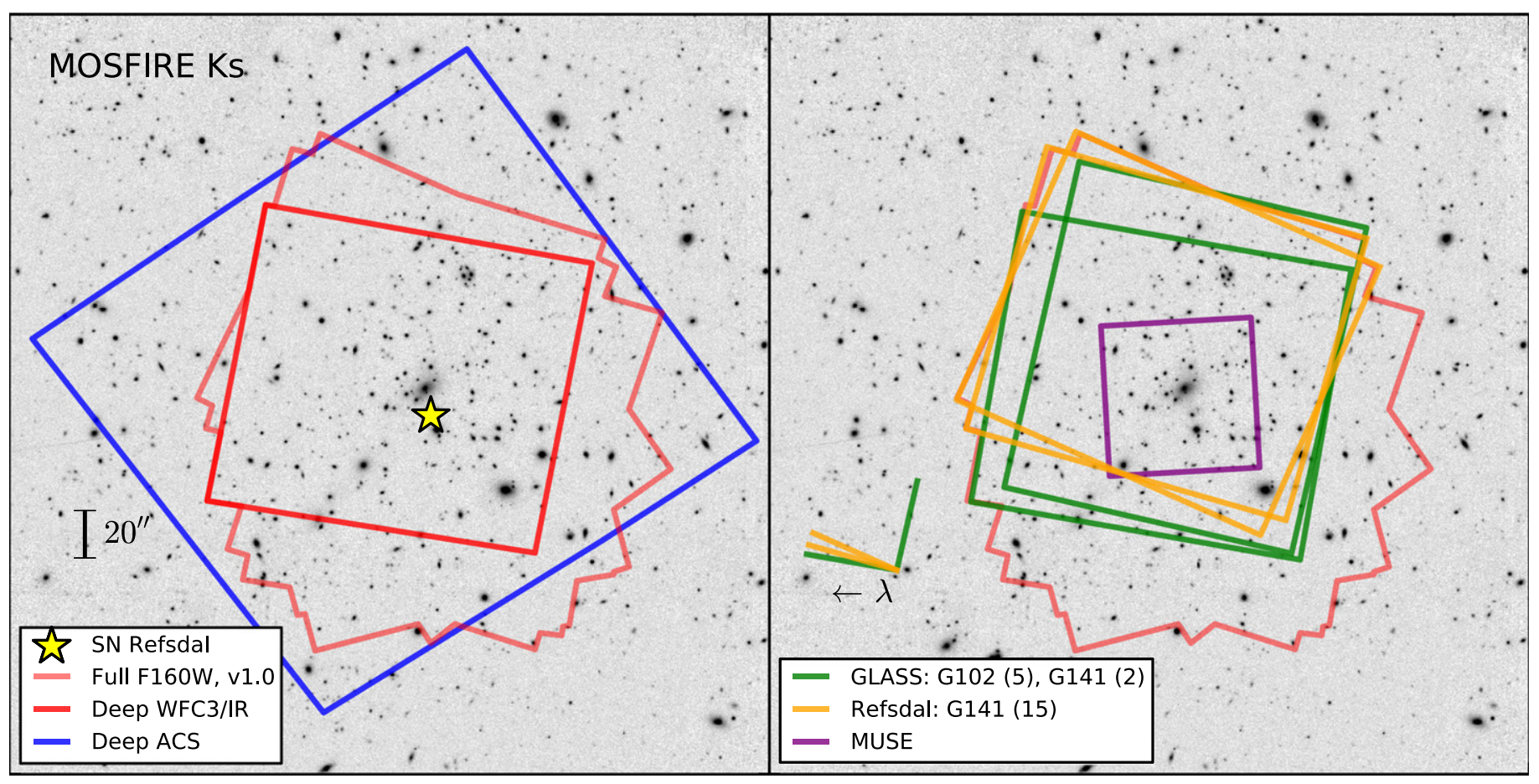

Figure 2. Observational layout of the MUSE and HST spectroscopy in the context of existing imaging data for MACSJ1149.5+2223. The "Full F160W" polygon is the full footprint of the F160W v1.0 FF release image. The numbers in parentheses in the spectroscopy panel at the right are the number of orbits per grism in each of two orientations. The background image was taken with the MOSFIRE instrument on the Keck I telescope (G. B. Brammer et al. 2015, in preparation).

Table 1

Measured Time Delays and Magnification Ratios

\begin{tabular}{|c|c|c|c|c|c|c|}
\hline \multirow[b]{2}{*}{ Image Pair } & \multicolumn{2}{|c|}{ SN Template (Prelim.) } & \multicolumn{2}{|c|}{ SN Template } & \multicolumn{2}{|c|}{ Polynomial } \\
\hline & $\begin{array}{c}\Delta \mathrm{t} \\
\text { (days) }\end{array}$ & $\mu$ ratio & $\begin{array}{c}\Delta \mathrm{t} \\
\text { (days) }\end{array}$ & $\mu$ ratio & $\begin{array}{c}\Delta \mathrm{t} \\
\text { (days) }\end{array}$ & $\mu$ ratio \\
\hline S3 S1 & $5.6 \pm 2.2$ & $1.04 \pm 0.02$ & $-0.9 \pm 1.1$ & $1.03 \pm 0.01$ & $-0.4_{-2.8}^{+1.9}$ & $1.01 \pm 0.02$ \\
\hline S4 S1 & $22 \pm 11$ & $0.35 \pm 0.01$ & $14.9 \pm 2.4$ & $0.34 \pm 0.03$ & $30.7_{-4.3}^{+4.8}$ & $0.38 \pm 0.01$ \\
\hline
\end{tabular}

Note. Observed delays and relative magnifications between images S1-S4 of SN Refsdal. For the values in columns 2 and 3, light curves extending up until 2015 July were used by one of us (P.K.) to derive time delays and magnification ratios using a range of templates. For the values in columns 4 and 5, Rodney et al. (2016) matched the observed S1-S4 light curves with the best available SN light-curve template, a model based on SN 1987A with corrections to account for the bluer color of SN Refsdal. The values in columns 6 and 7 were determined by fitting the HST photometry using a second-order Chebyshev polynomial.

uncertainty of \pm 20 days in the observer frame (Rodney et al. 2016).

The S1-S4 light curves enable a first measurement of the relative time delays and magnification ratios (Rodney et al. 2016). Preliminary results from that analysis, using light-curve data up until the end of 2015 July, were included in the first version of this paper, which was posted to the arxiv before October 30 in order to make a truly blind prediction before new observations could have revealed the reappearance of SN Refsdal at position SX. Those preliminary time delays were only used as a test of the models and not as an input to the model, so they did not affect any of the predictions given in this paper. Those preliminary measurements obtained by one of us (P.K.) using a range of SN templates are listed in Table 1 for reference. The preliminary peak date of $\mathrm{S} 1$ was found to be 2015 April 26 ( \pm 20 days), i.e., consistent with the final measurement given here (April 20) within the uncertainties. The final analysis by Rodney et al. (2015) incorporates Cycle
23 observations collected through 2015 November 14. In Section 5.2 we compare our model predictions against all three sets of available measurements of the S1-S4 time delays and magnifications. However, to preserve the blind test, the mass models in this work were not updated to accommodate new information from those late epochs (e.g., the peak is still assumed to be 2015 April 26 in the plots).

The Rodney et al. (2015) analysis uses two approaches for the time-delay measurements, first matching the best available SN template (based on SN 1987A) to each of the S1-S4 light curves, and then using a simple polynomial representation for the intrinsic light-curve shape. Uncertainties were derived using a mock light-curve algorithm similar to those developed for lensed quasars (Tewes et al. 2013a), although both measurements ignore effects like microlensing fluctuations (Dobler \& Keeton 2006), and therefore this should be considered as a lower limit to the total uncertainty. 
Results from these updated measurements are presented in Table 1 alongside the preliminary ones. The relative magnification ratios are measured to within $2 \%$, and consistent values are derived from both methods. However, the time delays inferred from the two approaches do not agree within the measured uncertainties. This is in part due to the slowly evolving, comparatively featureless light curve of SN Refsdal, and the fact that we were not able observe the MACSJ1149.5+2223 field in August through October, when the SN faded substantially from maximum light. The differences in inferred time delays may also reflect systematic biases inherent to one or both of the measurement methods, as it is possible that none of the available SN light-curve templates or the simple polynomial model are able to accurately represent the intrinsic light-curve shape of this peculiar event. The difference between the two sets of measurements provides an estimate of the systematic uncertainties.

\subsection{Spectroscopy}

\subsubsection{HST Spectroscopy}

The HST grism spectroscopy comprises two data sets. The GLASS data consist of 10 orbits of exposures taken through the G102 grism and 4 orbits of exposures taken through the G141 grism, spanning the wavelength range $0.81-1.69 \mu \mathrm{m}$. The GLASS data were taken at two approximately orthogonal position angles (PAs) to mitigate contamination by nearby sources (the first one on 2014 February 23-25, the second PA on 2014 November 3-11). The SN Refsdal follow-up effort was focused on the G141 grism, reaching a depth of 30 orbits. The pointing and PA of the follow-up grism data were chosen to optimize the spectroscopy of the SN itself, and are therefore different from the ones adopted by GLASS. The SN Refsdal follow-up spectra were taken between 2014 December 23 and 2015 January 4. Only a brief description of the data is given here. For more details the reader is referred to Schmidt et al. (2014) and Treu et al. (2015) for GLASS, and G. B. Brammer et al. (2015, in preparation) and P. L. Kelly et al. (2015, in preparation) for the deeper follow-up data.

The observing strategies and data-reduction schemes were very similar for the two data sets, building on previous work by the 3D-HST survey (Brammer et al. 2012). At least 4 subexposures were taken during each visit with semi-integer pixel offsets. This enables rejection of defects and cosmic rays as well as recovery of some of the resolution lost to undersampling of the point-spread function through interlacing. The data were reduced with an updated version of the 3D-HST reduction pipeline ${ }^{31}$ described by Brammer et al. (2012) and Momcheva et al. (2015). The pipeline takes care of alignment, defect removal, background removal, image combination, and modeling of contamination by nearby sources. One- and twodimensional spectra are extracted for each source.

The spectra were inspected independently by two of us (T.T. and G.B.) using custom tools and the interfaces $\mathrm{GiG}$ and $\mathrm{GiGz}$ (available at https://github.com/kasperschmidt/GLASSinspectionGUIs) developed as part of the GLASS project. Information obtained from the multiband photometry, continuum, and emission lines was combined to derive a redshift and quality flag. The few discrepancies between redshifts and quality flags were resolved by mutual agreement. In the end, we determined redshifts for 389 sources, with

\footnotetext{
${ }^{31}$ http://code.google.com/p/threedhst/
}

quality 3 or 4 (probable or secure, respectively, as defined by Treu et al. 2015).

\subsubsection{VLT Spectroscopy}

Integral-field spectroscopy was obtained with the MUSE instrument on the VLT between 2015 February 14 and 2015 April 12, as part of a Director Discretionary Time program to observe SN Refsdal (PI Grillo). The main goal of the program was to facilitate the computation of an accurate model to forecast the next appearance of the lensed SN. MUSE covers the wavelength range $480-930 \mathrm{~nm}$, with an average spectral resolution of $R \approx 3000$, over a $1^{\prime} \times 1^{\prime}$ field of view, with a pixel scale of $0{ }^{\prime \prime} 2 \mathrm{px}^{-1}$. Details of the data acquisition and processing are given in a separate paper (Grillo et al. 2015a); only a brief summary of relevant information is given here to guide the reader.

Twelve exposures were collected in dark time under clear conditions and with an average seeing of $\sim 1$ !'0. Bias subtraction, flatfielding, wavelength calibration, and flux calibration were obtained with the MUSE Data Reduction Software version 1.0, as described by Karman et al. (2015b, 2015a). The different exposures were combined into a single datacube, with a spectral sampling of $1.25 \AA \mathrm{px}^{-1}$, and a resulting total integration time of $4.8 \mathrm{hr}$. One-dimensional (1D) spectra within circular apertures of 0 ". 6 radius were extracted for all the objects visible in the coadded image along the spectral direction. We also searched in the datacube for faint emission-line galaxies that were not detected in the stacked image. Redshifts were first measured independently by two of the coauthors (W. K. and I. B.) and later reconciled in the very few cases with inconsistent estimates. The analysis yielded secure redshift values for 111 objects, of which 15 are multiple images of 6 different background sources.

\subsubsection{Keck Spectroscopy}

Spectroscopy of the field was obtained using the DEIMOS spectrograph (Faber et al. 2003) on the $10 \mathrm{~m}$ Keck II telescope on 2014 December 20. Conditions were acceptable, with photometric transparency and 1"! 3 seeing. The 600 line $\mathrm{mm}^{-1}$ grating was used, set to a central wavelength of $7200 \AA$, resulting in a scale of $0.65 \AA$ pixel $^{-1}$. A multi-slit mask of the field with $1^{\prime \prime}$ wide slits was observed for $7 \times 1600 \mathrm{~s}$ exposures. The SN images were the main targets, but a slit was also placed on image 4.1, yielding a measurement of its redshift, $z=2.951$, independent of but fully consistent with the results from VLT-MUSE. A full analysis of these data and subsequent Keck spectroscopy will be discussed elsewhere.

\subsubsection{Combined Redshift Catalog}

Redshifts for 70 objects were measured independently using both MUSE and GLASS data. We find that the redshifts of all objects in common agree within the uncertainties, attesting to the excellent quality of the data. The final redshift catalog, consisting of 429 entries, is given in electronic format in Table 2, and will be available through the GLASS public website at URL https://archive.stsci.edu/prepds/glass/ after the acceptance of this manuscript. We note that owing to the high resolution of the MUSE data, we improved the precision of the redshift of the SN Refsdal host galaxy to $z=1.489$ (cf. 1491 previously reported by Smith et al. 2009). Also, we revise the redshift of the multiply imaged source 3 with the new and 
Table 2

Redshift Catalog

\begin{tabular}{lcccccc}
\hline \hline $\mathrm{ID}^{\star}$ & $\begin{array}{c}\alpha \\
(\mathrm{J} 2000)\end{array}$ & $\begin{array}{c}\delta \\
(\mathrm{J} 2000)\end{array}$ & $z$ & quality & source & Notes \\
\hline 1 & 177.397188 & 22.393744 & 0.0000 & 4 & 2 & $\ldots$ \\
2 & 177.404017 & 22.403067 & 0.5660 & 4 & 2 & $\ldots$ \\
3 & 177.394525 & 22.400653 & 0.5410 & 4 & 2 & $\ldots$ \\
4 & 177.399663 & 22.399597 & 0.5360 & 4 & 2 & $\ldots$ \\
5 & 177.404054 & 22.392108 & 0.0000 & 4 & 2 & $\ldots$ \\
6 & 177.398554 & 22.389792 & 0.5360 & 4 & 2 & $\ldots$ \\
7 & 177.393010 & 22.396799 & 2.9490 & 4 & 4 & 4.1 \\
8 & 177.394400 & 22.400761 & 2.9490 & 4 & 2 & 4.2 \\
9 & 177.404192 & 22.406125 & 2.9490 & 4 & 2 & 4.3 \\
10 & 177.392904 & 22.404014 & 0.5140 & 4 & 2 & $\ldots$ \\
\hline
\end{tabular}

Note. First entries of the redshift catalog. The full catalog is given in its entirety in the electronic edition. The column "quality" contains the quality flag ( 3 = secure, 4 = probable). The column "source" gives the original source of the redshift: $1=H S T$, G. B. Brammer et al. (2015, in preparation); $2=$ MUSE, (Grillo et al. 2015a); $3=H S T+$ MUSE, $4=$ MUSE +Keck. The column "note" lists special comments about the object, e.g., if the object is part of a known multiple-image system.

(This table is available in its entirety in machine-readable form.)

reliable measurement $z=3.129$ based on unequivocal multiple line identifications ([O II] in the grism data, plus $\mathrm{Ly} \alpha$ in the MUSE data).

\section{SUMMARY OF LENS MODELING CONSTRAINTS}

\subsection{Multiple Images}

The strong lensing models that are considered in this paper use as constraints sets of multiply imaged lensed galaxies, as well as knots in the host galaxy of SN Refsdal. The five teams independently evaluated known sets of multiple images (Smith et al. 2009; Zitrin \& Broadhurst 2009; Johnson et al. 2014; Diego et al. 2015; Sharon \& Johnson 2015), and suggested new identifications of images across the entire field of view, based on the new HFF data. In evaluating the image identifications, the teams relied on their preliminary lens models and the newly measured spectroscopic redshifts (Section 2.2). Each team voted on known and new systems on a scale of 1-4, where 1 denotes secure identification, 2 is a possible identification, and higher values are considered unreliable. Images that had large variance in their scores were discussed and reevaluated, and the final score was then recorded. The list of multiple images considered in this work is given in Table 3. For each system we give coordinates, the average score, and the redshift if available. We also indicate the labels given to known images that were previously identified in the literature, previously published redshifts, and references to these publications.

We define three samples of image sets ("gold," "silver," and "all") based on the voting process. Following the approach of Wang et al. (2015), we conservatively include in our gold sample only the systems about which every team was confident. The silver sample includes images that were considered secure by most teams, or are outside the MUSE field of view. The "all" sample includes all of the images that were not rejected as false identifications, based on imaging and/or spectroscopy. In order to facilitate the comparison, most teams produced baseline models based on the gold sample of images, and some of the teams produced additional models based on larger sets of images. However, owing to differences in investigators' opinions and specifics of each code, small differences between the constraints adopted by each team persist. They are described below for each of the teams. The reader is referred to the publications of each individual team for more details.

We also evaluated the identification of knots in the spiral galaxy hosting SN Refsdal. Table 4 and Figure 3 list the emission knots and features in the host galaxy of SN Refsdal that were considered in this work. Not all knots were used in all models, and again, there are slight differences between the teams as the implementation of these constraints vary among lensing algorithms. Nevertheless, the overall mapping of morphological features between the images of this galaxy was in agreement between the modeling teams.

\subsection{Time Delays}

The time delay and magnification ratios between the known images were not yet measured at the time when the models were being finalized. Therefore, they were not used as input and they can be considered as a valuable test of the lens model.

\subsection{Cluster Members}

Cluster member galaxies were selected based on their redshifts in the combined redshift catalog and their photometry, as follows. In order to account for the cluster velocity dispersion, as well as the uncertainty in the grism-based redshifts, we define cluster membership loosely as galaxies with spectroscopic redshift in the range $0.520<z<0.570$, within a few thousand kilometers per second of the fiducial cluster redshift $\left(z_{\mathrm{d}}=0.542\right)$. This is sufficiently precise for the purpose of building lens models, even though not all the cluster members are necessarily physically bound to the cluster, from a dynamical point of view. Naturally, these cluster members still contribute to the deflection field as the dynamically bound cluster members. The spectroscopic cluster-member catalog comprises 170 galaxies.

To obtain a more complete member catalog, the spectroscopically confirmed members were supplemented by photometrically selected galaxies. This list includes galaxies down to the limit ( $\mathrm{F} 814 \mathrm{~W} \approx 25 \mathrm{mag}$ ) of spectroscopically confirmed members. It consists mostly of galaxies belonging to the last two-magnitude bins of the luminosity distribution, for which the spectroscopic sample is significantly incomplete. The missing galaxies from the spectroscopic catalog are the brightest ones that fall outside the MUSE field of view or the ones that are contaminated in the HST grism data. The photometric analysis is restricted to the WFC3-IR area, in order to exploit the full multi-band photometric catalog from CLASH. The method is briefly described by Grillo et al. (2015b), and it uses a Bayesian technique to compute the probability for a galaxy to be a member from the distribution in color space of all spectroscopic galaxies (from 13 bands-i.e., not including the 3 in the UV). For the photometric selection, we started from spectroscopically confirmed members, with redshift within $0.520<z<0.570$, and provided a catalog with only the objects having measured F160W magnitudes. The total catalog of cluster members comprises 170 galaxies with spectroscopically determined membership, and 136 galaxies with photometrically determined membership. 
Table 3

Multiply Imaged Systems

\begin{tabular}{|c|c|c|c|c|c|c|c|c|c|c|c|c|c|}
\hline ID & $\begin{array}{c}\alpha \\
(\mathrm{J} 2000)\end{array}$ & $\begin{array}{c}\delta \\
(\mathrm{J} 2000)\end{array}$ & 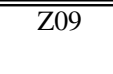 & S09 & $\begin{array}{c}\text { R14, } \\
\text { J14 }\end{array}$ & $\overline{\mathrm{D} 15}$ & 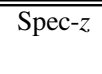 & $\overline{\text { Ref }}$ & Spec- $z$ & Source & Notes & $\begin{array}{l}\text { Avg. } \\
\text { Score }\end{array}$ & Category \\
\hline 1.1 & 177.39700 & 22.396000 & 1.2 & A1.1 & 1.1 & 1.1 & 1.4906 & S09 & 1.488 & 3 & $\ldots$ & 1.0 & Gold \\
\hline 1.2 & 177.39942 & 22.397439 & 1.3 & A1.2 & 1.2 & 1.2 & 1.4906 & S09 & 1.488 & 3 & $\cdots$ & 1.0 & Gold \\
\hline 1.3 & 177.40342 & 22.402439 & 1.1 & A 1.3 & 1.3 & 1.3 & 1.4906 & S09 & 1.488 & 3 & $\ldots$ & 1.0 & Gold \\
\hline 1.5 & 177.39986 & 22.397133 & 1.4 & $\ldots$ & $\ldots$ & 1.5 & $\ldots$ & $\ldots$ & $\ldots$ & $\ldots$ & 1 & 2.0 & $\ldots$ \\
\hline 2.1 & 177.40242 & 22.389750 & 3.3 & A2.1 & 2.1 & 2.3 & 1.894 & S09 & 1.891 & 3 & $\cdots$ & 1.0 & Gold \\
\hline 2.2 & 177.40604 & 22.392478 & 3.2 & A 2.2 & 2.2 & 2.2 & 1.894 & S09 & 1.891 & 3 & $\cdots$ & 1.0 & Gold \\
\hline 2.3 & 177.40658 & 22.392886 & 3.1 & A 2.3 & 2.3 & 2.1 & 1.894 & S09 & 1.891 & 3 & $\ldots$ & 1.0 & Gold \\
\hline 3.1 & 177.39075 & 22.399847 & 2.1 & A3.1 & 3.1 & 3.1 & 2.497 & S09 & 3.129 & 3 & 2 & 1.0 & Gold \\
\hline 3.2 & 177.39271 & 22.403081 & 2.2 & A 3.2 & 3.2 & 3.2 & 2.497 & S09 & 3.129 & 3 & 2 & 1.0 & Gold \\
\hline 3.3 & 177.40129 & 22.407189 & 2.3 & A 3.3 & 3.3 & 3.3 & $\ldots$ & $\ldots$ & 3.129 & 3 & 2 & 1.1 & Gold \\
\hline 4.1 & 177.39300 & 22.396825 & 4.1 & $\ldots$ & 4.1 & 4.1 & $\ldots$ & $\ldots$ & 2.949 & 4 & $\ldots$ & 1.0 & Gold \\
\hline 4.2 & 177.39438 & 22.400736 & 4.2 & $\ldots$ & 4.2 & 4.2 & $\ldots$ & $\ldots$ & 2.949 & 2 & $\ldots$ & 1.0 & Gold \\
\hline 4.3 & 177.40417 & 22.406128 & 4.3 & $\ldots$ & 4.3 & 4.3 & $\ldots$ & $\ldots$ & 2.949 & 2 & $\ldots$ & 1.0 & Gold \\
\hline 5.1 & 177.39975 & 22.393061 & 5.1 & $\ldots$ & 5.1 & 5.1 & $\ldots$ & $\ldots$ & 2.80 & 1 & $\ldots$ & 1.0 & Gold \\
\hline 5.2 & 177.40108 & 22.393825 & 5.2 & $\ldots$ & 5.2 & 5.2 & $\ldots$ & $\ldots$ & $\ldots$ & $\ldots$ & $\ldots$ & 1.0 & Gold \\
\hline 5.3 & 177.40792 & 22.403553 & 5.3 & $\ldots$ & 5.3 & $\ldots$ & $\ldots$ & $\ldots$ & $\ldots$ & $\ldots$ & $\ldots$ & 1.7 & Silver \\
\hline 6.1 & 177.39971 & 22.392544 & 6.1 & $\ldots$ & 6.1 & 6.1 & $\cdots$ & $\ldots$ & $\ldots$ & $\ldots$ & $\ldots$ & 1.1 & Gold \\
\hline 6.2 & 177.40183 & 22.393858 & 6.2 & $\ldots$ & 6.2 & 6.2 & $\cdots$ & $\cdots$ & $\cdots$ & $\ldots$ & $\cdots$ & 1.1 & Gold \\
\hline 6.3 & 177.40804 & 22.402506 & $5.4 / 6.3$ & $\cdots$ & 6.3 & $\ldots$ & $\cdots$ & $\ldots$ & $\ldots$ & $\ldots$ & $\cdots$ & 1.7 & Silver \\
\hline 7.1 & 177.39896 & 22.391339 & 7.1 & $\ldots$ & 7.1 & $\ldots$ & $\ldots$ & $\ldots$ & $\ldots$ & $\ldots$ & $\ldots$ & 1.1 & Gold \\
\hline 7.2 & 177.40342 & 22.394269 & 7.2 & $\ldots$ & 7.2 & $\ldots$ & $\ldots$ & $\ldots$ & $\ldots$ & $\ldots$ & $\ldots$ & 1.1 & Gold \\
\hline 7.3 & 177.40758 & 22.401242 & $\ldots$ & $\ldots$ & 7.3 & $\ldots$ & $\ldots$ & $\ldots$ & $\ldots$ & $\ldots$ & $\ldots$ & 1.2 & Gold \\
\hline 8.1 & 177.39850 & 22.394350 & 8.1 & $\ldots$ & 8.1 & 8.1 & $\ldots$ & $\ldots$ & $\ldots$ & $\ldots$ & $\ldots$ & 1.2 & Gold \\
\hline 8.2 & 177.39979 & 22.395044 & 8.2 & $\ldots$ & 8.2 & 8.2 & $\ldots$ & $\ldots$ & $\ldots$ & $\ldots$ & $\ldots$ & 1.2 & Gold \\
\hline 8.4 & 177.40709 & 22.404722 & $\ldots$ & $\ldots$ & $\ldots$ & $\ldots$ & $\ldots$ & $\ldots$ & $\ldots$ & $\ldots$ & 3 & 1.2 & Gold \\
\hline$\cdots$ & 177.40704 & 22.405553 & $\cdots$ & $\ldots$ & $\ldots$ & $\cdots$ & $\cdots$ & $\ldots$ & 2.78 & 1 & 3 & 3.0 & Rejected \\
\hline$\cdots$ & 177.40517 & 22.401563 & 8.3 & $\cdots$ & $\cdots$ & $\cdots$ & $\cdots$ & $\cdots$ & $\cdots$ & $\cdots$ & 3 & 3.0 & Rejected \\
\hline 9.1 & 177.40517 & 22.426233 & $\ldots$ & A6.1 & 9.1 & $\ldots$ & $\ldots$ & $\ldots$ & $\ldots$ & $\ldots$ & $\ldots$ & 1.8 & $\ldots$ \\
\hline 9.2 & 177.40388 & 22.427231 & $\ldots$ & A6.2 & 9.2 & $\ldots$ & $\ldots$ & $\ldots$ & $\ldots$ & $\ldots$ & $\ldots$ & 1.8 & $\ldots$ \\
\hline 9.3 & 177.40325 & 22.427228 & $\ldots$ & A6.3 & 9.3 & $\ldots$ & $\ldots$ & $\ldots$ & $\ldots$ & $\ldots$ & $\ldots$ & 1.8 & $\ldots$ \\
\hline 9.4 & 177.40364 & 22.426422 & $\ldots$ & A6.4? & $\ldots$ & $\ldots$ & $\ldots$ & $\ldots$ & $\ldots$ & $\ldots$ & $\ldots$ & 1.8 & $\ldots$ \\
\hline 10.1 & 177.40450 & 22.425514 & $\ldots$ & A7.1 & 10.1 & $\ldots$ & $\ldots$ & $\ldots$ & $\ldots$ & $\ldots$ & $\ldots$ & 1.8 & $\ldots$ \\
\hline 10.2 & 177.40362 & 22.425636 & $\cdots$ & A7.2 & 10.2 & $\cdots$ & $\ldots$ & $\cdots$ & $\cdots$ & $\cdots$ & $\cdots$ & 1.8 & $\cdots$ \\
\hline 10.3 & 177.40221 & 22.426625 & $\ldots$ & A7.3 & 10.3 & $\ldots$ & $\ldots$ & $\ldots$ & $\ldots$ & $\ldots$ & $\ldots$ & 1.8 & $\ldots$ \\
\hline 12.1 & 177.39857 & 22.389356 & $\ldots$ & $\ldots$ & $\ldots$ & $\ldots$ & $\ldots$ & $\ldots$ & 1.020 & 3 & 4 & 2.6 & Rejected \\
\hline 12.2 & 177.40375 & 22.392345 & $\ldots$ & $\ldots$ & $\ldots$ & $\ldots$ & $\ldots$ & $\ldots$ & 0.929 & 2 & 4 & 2.9 & Rejected \\
\hline 12.3 & 177.40822 & 22.398801 & $\cdots$ & $\cdots$ & $\ldots$ & $\cdots$ & $\cdots$ & $\cdots$ & 1.118 & 3 & 4 & 2.6 & Rejected \\
\hline 13.1 & 177.40371 & 22.397786 & $\ldots$ & $\ldots$ & 13.1 & $\ldots$ & $\cdots$ & $\cdots$ & 1.23 & 1 & $\cdots$ & 1.0 & Gold \\
\hline 13.2 & 177.40283 & 22.396656 & $\ldots$ & $\ldots$ & 13.2 & $\ldots$ & $\ldots$ & $\ldots$ & 1.25 & 1 & $\ldots$ & 1.0 & Gold \\
\hline 13.3 & 177.40004 & 22.393858 & $\ldots$ & $\ldots$ & 13.3 & $\ldots$ & $\ldots$ & $\ldots$ & 1.23 & 1 & $\ldots$ & 1.3 & Gold \\
\hline 14.1 & 177.39167 & 22.403489 & $\ldots$ & $\ldots$ & 14.1 & $\ldots$ & $\ldots$ & $\ldots$ & 3.703 & 2 & $\cdots$ & 1.3 & Gold \\
\hline 14.2 & 177.39083 & 22.402647 & $\ldots$ & $\ldots$ & 14.2 & $\ldots$ & $\ldots$ & $\ldots$ & 3.703 & 2 & $\ldots$ & 1.3 & Gold \\
\hline 110.1 & 177.40014 & 22.390162 & $\ldots$ & $\ldots$ & $\ldots$ & $\ldots$ & $\ldots$ & $\ldots$ & 3.214 & 2 & $\ldots$ & 1.0 & Gold \\
\hline 110.2 & 177.40402 & 22.392894 & $\ldots$ & $\ldots$ & $\ldots$ & $\ldots$ & $\ldots$ & $\ldots$ & 3.214 & 2 & $\ldots$ & 1.0 & Gold \\
\hline 110.3 & 177.40907 & 22.400242 & $\ldots$ & $\ldots$ & $\ldots$ & $\ldots$ & $\ldots$ & $\ldots$ & $\ldots$ & $\ldots$ & $\cdots$ & 2.0 & $\ldots$ \\
\hline 21.1 & 177.40451 & 22.386704 & $\ldots$ & $\ldots$ & $\ldots$ & $\ldots$ & $\ldots$ & $\ldots$ & $\ldots$ & $\ldots$ & $\ldots$ & 1.8 & Silver \\
\hline 21.2 & 177.40800 & 22.389057 & $\ldots$ & $\ldots$ & $\ldots$ & $\ldots$ & $\ldots$ & $\ldots$ & $\ldots$ & $\ldots$ & $\cdots$ & 1.6 & Silver \\
\hline 21.3 & 177.40907 & 22.390407 & $\ldots$ & $\ldots$ & $\ldots$ & $\ldots$ & $\ldots$ & $\ldots$ & $\ldots$ & $\ldots$ & $\ldots$ & 1.6 & Silver \\
\hline 22.1 & 177.40370 & 22.386838 & $\ldots$ & $\ldots$ & $\ldots$ & $\ldots$ & $\ldots$ & $\ldots$ & $\ldots$ & $\ldots$ & $\cdots$ & 1.8 & $\ldots$ \\
\hline 22.2 & 177.40791 & 22.389232 & $\ldots$ & $\ldots$ & $\ldots$ & $\ldots$ & $\ldots$ & $\ldots$ & $\ldots$ & $\ldots$ & $\ldots$ & 1.8 & $\ldots$ \\
\hline 22.3 & 177.40902 & 22.391053 & $\ldots$ & $\ldots$ & $\ldots$ & $\ldots$ & $\ldots$ & $\ldots$ & $\ldots$ & $\ldots$ & $\ldots$ & 1.8 & $\ldots$ \\
\hline 23.1 & 177.39302 & 22.411428 & $\ldots$ & A5 & $\ldots$ & $\ldots$ & $\ldots$ & $\ldots$ & $\ldots$ & $\ldots$ & $\ldots$ & 1.8 & $\ldots$ \\
\hline 23.2 & 177.39308 & 22.411455 & $\ldots$ & A5 & $\ldots$ & $\ldots$ & $\ldots$ & $\ldots$ & $\ldots$ & $\ldots$ & $\ldots$ & 1.8 & $\ldots$ \\
\hline 23.3 & 177.39315 & 22.411473 & $\ldots$ & A5 & $\ldots$ & $\ldots$ & $\ldots$ & $\ldots$ & $\ldots$ & $\ldots$ & $\ldots$ & 1.8 & $\ldots$ \\
\hline 24.1 & 177.39285 & 22.412872 & $\ldots$ & $\ldots$ & $\ldots$ & $\cdots$ & $\ldots$ & $\cdots$ & $\cdots$ & $\cdots$ & $\ldots$ & 1.7 & $\ldots$ \\
\hline 24.2 & 177.39353 & 22.413071 & $\ldots$ & $\ldots$ & $\ldots$ & $\ldots$ & $\ldots$ & $\ldots$ & $\ldots$ & $\ldots$ & $\ldots$ & 1.7 & $\ldots$ \\
\hline 24.3 & 177.39504 & 22.412697 & $\ldots$ & $\ldots$ & $\ldots$ & $\ldots$ & $\ldots$ & $\ldots$ & $\ldots$ & $\ldots$ & $\ldots$ & 1.8 & $\ldots$ \\
\hline 25.1 & 177.40428 & 22.398782 & $\ldots$ & $\ldots$ & $\ldots$ & $\ldots$ & $\ldots$ & $\ldots$ & $\ldots$ & $\ldots$ & $\ldots$ & 2.0 & $\ldots$ \\
\hline 25.2 & 177.40411 & 22.398599 & $\ldots$ & $\ldots$ & $\ldots$ & $\ldots$ & $\ldots$ & $\ldots$ & $\ldots$ & $\ldots$ & $\ldots$ & 2.0 & $\ldots$ \\
\hline 25.3 & 177.39489 & 22.391796 & $\ldots$ & $\ldots$ & $\ldots$ & $\ldots$ & $\ldots$ & $\ldots$ & $\ldots$ & $\ldots$ & $\ldots$ & 2.3 & $\ldots$ \\
\hline 26.1 & 177.41035 & 22.388749 & 9.1 & $\ldots$ & $\ldots$ & $\ldots$ & $\ldots$ & $\ldots$ & $\ldots$ & $\ldots$ & $\cdots$ & 1.8 & Silver \\
\hline 26.2 & 177.40922 & 22.387697 & 9.2 & $\ldots$ & $\ldots$ & $\cdots$ & $\cdots$ & $\ldots$ & $\cdots$ & $\ldots$ & $\cdots$ & 1.8 & Silver \\
\hline 26.3 & 177.40623 & 22.385369 & $\ldots$ & $\ldots$ & $\ldots$ & $\ldots$ & $\ldots$ & $\ldots$ & $\ldots$ & $\ldots$ & $\ldots$ & 1.8 & Silver \\
\hline
\end{tabular}


Table 3

(Continued)

\begin{tabular}{|c|c|c|c|c|c|c|c|c|c|c|c|c|c|}
\hline 27.1 & 177.40971 & 22.387665 & $\cdots$ & $\cdots$ & $\cdots$ & $\cdots$ & $\cdots$ & $\cdots$ & $\cdots$ & $\cdots$ & $\cdots$ & 1.8 & Silver \\
\hline 27.2 & 177.40988 & 22.387835 & $\ldots$ & $\ldots$ & $\cdots$ & $\cdots$ & $\ldots$ & $\ldots$ & $\ldots$ & $\ldots$ & $\cdots$ & 1.8 & Silver \\
\hline 27.3 & 177.40615 & 22.385142 & $\ldots$ & $\ldots$ & $\ldots$ & $\ldots$ & $\ldots$ & $\ldots$ & $\ldots$ & $\ldots$ & $\ldots$ & 2.5 & $\ldots$ \\
\hline 28.1 & 177.39531 & 22.391809 & $\cdots$ & $\cdots$ & $\cdots$ & $\cdots$ & $\cdots$ & $\cdots$ & $\cdots$ & $\cdots$ & $\ldots$ & 2.0 & $\ldots$ \\
\hline 28.2 & 177.40215 & 22.396750 & $\cdots$ & $\cdots$ & $\cdots$ & $\cdots$ & $\cdots$ & $\cdots$ & $\cdots$ & $\cdots$ & $\cdots$ & 2.2 & $\cdots$ \\
\hline 28.3 & 177.40562 & 22.402434 & $\ldots$ & $\ldots$ & $\cdots$ & $\ldots$ & $\ldots$ & $\ldots$ & $\ldots$ & $\ldots$ & $\ldots$ & 2.0 & $\ldots$ \\
\hline 200.1 & 177.40875 & 22.394467 & $\cdots$ & $\cdots$ & $\cdots$ & $\cdots$ & $\cdots$ & $\ldots$ & 2.32 & 1 & $\ldots$ & 2.6 & $\cdots$ \\
\hline 200.2 & 177.40512 & 22.391261 & $\cdots$ & $\cdots$ & $\cdots$ & $\cdots$ & $\cdots$ & $\cdots$ & $\ldots$ & $\cdots$ & $\cdots$ & 2.6 & $\cdots$ \\
\hline 200.3 & 177.40256 & 22.389233 & $\cdots$ & $\cdots$ & $\cdots$ & $\cdots$ & $\cdots$ & $\cdots$ & $\cdots$ & $\cdots$ & $\ldots$ & 2.8 & $\cdots$ \\
\hline 201.1 & 177.40048 & 22.395444 & $\cdots$ & $\cdots$ & $\cdots$ & $\cdots$ & $\cdots$ & $\ldots$ & $\cdots$ & $\ldots$ & 5 & 1.6 & $\ldots$ \\
\hline 201.2 & 177.40683 & 22.404517 & $\ldots$ & $\cdots$ & $\ldots$ & $\ldots$ & $\cdots$ & $\ldots$ & $\cdots$ & $\ldots$ & 5 & 1.6 & $\ldots$ \\
\hline 202.1 & 177.40765 & 22.396789 & $\cdots$ & $\cdots$ & $\cdots$ & $\cdots$ & $\cdots$ & $\cdots$ & $\cdots$ & $\cdots$ & $\cdots$ & 2.0 & $\cdots$ \\
\hline 202.2 & 177.40224 & 22.391489 & $\ldots$ & $\ldots$ & $\cdots$ & $\ldots$ & $\ldots$ & $\ldots$ & $\cdots$ & $\ldots$ & $\cdots$ & 2.0 & $\ldots$ \\
\hline 202.3 & 177.40353 & 22.392586 & $\cdots$ & $\cdots$ & $\cdots$ & $\cdots$ & $\cdots$ & $\cdots$ & $\cdots$ & $\ldots$ & $\ldots$ & 2.0 & $\ldots$ \\
\hline 203.1 & 177.40995 & 22.387244 & $\cdots$ & $\cdots$ & $\cdots$ & $\cdots$ & $\cdots$ & $\cdots$ & $\cdots$ & $\cdots$ & $\ldots$ & 1.8 & Silver \\
\hline 203.2 & 177.40657 & 22.384511 & $\ldots$ & $\cdots$ & $\cdots$ & $\cdots$ & $\cdots$ & $\cdots$ & $\cdots$ & $\cdots$ & $\cdots$ & 2.0 & Silver \\
\hline 203.3 & 177.41123 & 22.388461 & $\cdots$ & $\cdots$ & $\cdots$ & $\cdots$ & $\cdots$ & $\cdots$ & $\cdots$ & $\cdots$ & $\cdots$ & 1.8 & Silver \\
\hline 204.1 & 177.40961 & 22.386661 & $\ldots$ & $\ldots$ & $\cdots$ & $\ldots$ & $\ldots$ & $\ldots$ & $\ldots$ & $\ldots$ & $\ldots$ & 1.8 & Silver \\
\hline 204.2 & 177.40668 & 22.384322 & $\cdots$ & $\cdots$ & $\cdots$ & $\ldots$ & $\cdots$ & $\ldots$ & $\cdots$ & $\cdots$ & $\ldots$ & 1.8 & Silver \\
\hline 204.3 & 177.41208 & 22.389056 & $\cdots$ & $\cdots$ & $\cdots$ & $\cdots$ & $\cdots$ & $\cdots$ & $\cdots$ & $\cdots$ & $\cdots$ & 1.8 & Silver \\
\hline 205.1 & 177.40520 & 22.386042 & $\cdots$ & $\cdots$ & $\cdots$ & $\cdots$ & $\cdots$ & $\cdots$ & $\cdots$ & $\cdots$ & $\cdots$ & 2.0 & $\ldots$ \\
\hline 205.2 & 177.40821 & 22.388119 & $\cdots$ & $\cdots$ & $\cdots$ & $\ldots$ & $\cdots$ & $\ldots$ & $\cdots$ & $\cdots$ & $\cdots$ & 2.0 & $\cdots$ \\
\hline 205.3 & 177.41038 & 22.390625 & $\cdots$ & $\cdots$ & $\cdots$ & $\cdots$ & $\cdots$ & $\cdots$ & $\cdots$ & $\cdots$ & $\cdots$ & 2.0 & $\cdots$ \\
\hline 206.1 & 177.40764 & 22.385647 & $\cdots$ & $\cdots$ & $\cdots$ & $\cdots$ & $\cdots$ & $\cdots$ & $\cdots$ & $\cdots$ & $\cdots$ & 2.2 & $\cdots$ \\
\hline 206.2 & 177.40863 & 22.386453 & $\ldots$ & $\ldots$ & $\ldots$ & $\cdots$ & $\cdots$ & $\cdots$ & $\cdots$ & $\cdots$ & $\ldots$ & 2.2 & $\ldots$ \\
\hline 206.3 & 177.41133 & 22.388997 & $\cdots$ & $\cdots$ & $\cdots$ & $\cdots$ & $\cdots$ & $\cdots$ & $\cdots$ & $\cdots$ & $\cdots$ & 2.2 & $\cdots$ \\
\hline 207.1 & 177.40442 & 22.397303 & $\ldots$ & $\cdots$ & $\cdots$ & $\ldots$ & $\cdots$ & $\cdots$ & $\cdots$ & $\ldots$ & $\ldots$ & 2.2 & $\ldots$ \\
\hline 207.2 & 177.40397 & 22.396039 & $\cdots$ & $\cdots$ & $\cdots$ & $\cdots$ & $\cdots$ & $\cdots$ & $\cdots$ & $\cdots$ & $\cdots$ & 2.2 & $\cdots$ \\
\hline 208.1 & 177.40453 & 22.395761 & $\cdots$ & $\cdots$ & $\cdots$ & $\cdots$ & $\cdots$ & $\cdots$ & $\cdots$ & $\cdots$ & $\cdots$ & 2.0 & $\cdots$ \\
\hline 208.2 & 177.40494 & 22.396397 & $\ldots$ & $\ldots$ & $\cdots$ & $\ldots$ & $\ldots$ & $\ldots$ & $\ldots$ & $\ldots$ & $\ldots$ & 2.0 & $\ldots$ \\
\hline 209.1 & 177.38994 & 22.412694 & $\ldots$ & $\ldots$ & $\ldots$ & $\ldots$ & $\ldots$ & $\ldots$ & $\ldots$ & $\ldots$ & $\ldots$ & 3.0 & $\ldots$ \\
\hline 209.2 & 177.39055 & 22.413408 & $\cdots$ & $\cdots$ & $\cdots$ & $\cdots$ & $\cdots$ & $\cdots$ & $\cdots$ & $\cdots$ & $\cdots$ & 3.0 & $\cdots$ \\
\hline 210.1 & 177.39690 & 22.398061 & $\ldots$ & $\ldots$ & $\cdots$ & $\ldots$ & $\cdots$ & $\cdots$ & 0.702 & 2 & $\ldots$ & 3.0 & $\ldots$ \\
\hline 210.2 & 177.39505 & 22.397497 & $\cdots$ & $\cdots$ & $\cdots$ & $\cdots$ & $\cdots$ & $\cdots$ & 0.702 & 2 & $\cdots$ & 3.0 & $\cdots$ \\
\hline
\end{tabular}

Notes. Coordinates and ID notations of multiply imaged families of lensed galaxies. The labels in previous publications are indicated for Zitrin et al. (2009; Z09), Smith et al. (2009; S09), Richard et al. (2014; R14), Johnson et al. (2014; J14), and Diego et al. (2015; D15). New identifications were made by Sharon, Oguri, and Hoag. Each modeling team used a modified version or subset of the list above, with the coordinates of each knot varying slightly between modelers. The source of the new spectroscopic redshift is as in Table 2: $1=H S T$, G. B. Brammer et al. (2015, in preparation); $2=$ MUSE, (Grillo et al. 2015a); $3=H S T+$ MUSE; $4=$ MUSE + Keck. The redshift of image 4.1 was measured independently at Keck (Section 2.2.3). The average score among the team is recorded; " 1 " denotes secure identification, " 2 " is a possible identification, and higher scores are considered unreliable by the teams. (1) See Table 4 for information on all the knots in source 1. (2) We revise the redshift of source 3 with the new and reliable measurement from MUSE (see Section 2.2). (3) We revise the identification of a counterimage of 8.1 and 8.2, and determine that it is at a different position compared to previous publications. To limit confusion we label the newly identified counterimage 8.4. (4) The identification of source 12 was ruled out in HFF work prior to the 2014 publications; we further reject this set with spectroscopy. (5) This image is identified as part of the same source as source 8 ; the third image is buried in the light of a nearby star.

\section{BRIEF DESCRIPTION OF MODELING TECHNIQUES AND THEIR INPUTS}

For convenience to the reader, in this section we give a brief description of each of the modeling techniques compared in this work (summarized briefly in Table 5). We note that the five models span a range of very different assumptions. Three of the teams (Grillo et al., Oguri et al., Sharon et al.) used an approach based on modeling the mass distribution with a set of physically motivated components, described each by a small number of parameters, representing the galaxies in the cluster and the overall cluster halo. We refer to these models as "simply parametrized." One of the approaches (Diego et al.) describes the mass distribution with a larger number of

\footnotetext{
32 These models are sometimes described incorrectly as "nonparametric," even though they typically have more parameters than the so-called parametric models.
}

components. The components are not associated with any specific physical object and are used as building blocks, allowing for significant flexibility, balanced by regularization. We refer to this model as "free-form." ${ }^{32}$ The fifth approach (Zitrin et al.) is based on the assumption that light approximately traces mass, and the mass components are built by smoothing and rescaling the observed surface brightness of the cluster members. We refer to this approach as "light-tracesmass." All of the models considered here are single-plane lens models. As we will discuss in Section 6, each type of model uses a different approach to account for the effects of structure along the line of sight, and to break the mass-sheet degeneracy. All model outputs will be made available through the HFF website after the acceptance of the individual modeling papers.

We note that members of our team have developed another complementary "free-form" approach, based on modeling the potential in pixels on an adaptive grid 
Table 4

Knots in the Host Galaxy of SN Refsdal

\begin{tabular}{|c|c|c|c|c|c|c|}
\hline ID & $\alpha(\mathrm{J} 2000)$ & $\delta(\mathrm{J} 2000)$ & $\begin{array}{l}\text { ID } \\
\text { Smith } \\
\text { et al. } \\
(2009)\end{array}$ & $\begin{array}{c}\text { ID } \\
\text { K. Sharon } \\
\text { et al. (2016, in } \\
\text { preparation) }\end{array}$ & $\begin{array}{c}\text { ID } \\
\text { Diego } \\
\text { et al. } \\
(2015)\end{array}$ & Notes \\
\hline 1.1 .1 & 177.39702 & 22.396003 & 2 & 1.1 & 1.1 .1 & 1 \\
\hline 1.1 .2 & 177.39942 & 22.397439 & 2 & 1.2 & 1.2 .1 & 1 \\
\hline 1.1.3 & 177.40341 & 22.402444 & 2 & 1.3 & 1.3 .1 & 1 \\
\hline 1. ${ }^{*} 1.5$ & 177.39986 & 22.397133 & $\ldots$ & $\ldots$ & 1.5 .1 & 1,2 \\
\hline 1.2 .1 & 177.39661 & 22.396308 & 19 & 23.1 & 1.1 .8 & $\ldots$ \\
\hline 1.2 .2 & 177.39899 & 22.397867 & 19 & 23.2 & 1.2 .8 & $\ldots$ \\
\hline 1.2 .3 & 177.40303 & 22.402681 & 19 & 23.3 & 1.3 .8 & $\cdots$ \\
\hline 1.2 .4 & 177.39777 & 22.398789 & 19 & 23.4 & $1.4 .8 \mathrm{a}$ & $\ldots$ \\
\hline 1.2 .6 & 177.39867 & 22.398242 & $\ldots$ & $\ldots$ & $1.4 .8 \mathrm{~b}$ & $\ldots$ \\
\hline 1.3.1 & 177.39687 & 22.396219 & 16 & 31.1 & 1.1 .15 & $\cdots$ \\
\hline 1.3 .2 & 177.39917 & 22.397600 & 16 & 31.2 & 1.2 .15 & $\cdots$ \\
\hline 1.3 .3 & 177.40328 & 22.402594 & 16 & 31.3 & 1.3 .15 & $\ldots$ \\
\hline 1.4 .1 & 177.39702 & 22.396214 & 11 & 32.1 & $\ldots$ & $\ldots$ \\
\hline 1.4 .2 & 177.39923 & 22.397483 & 11 & 32.2 & $\ldots$ & $\cdots$ \\
\hline 1.4 .3 & 177.40339 & 22.402558 & 11 & 32.3 & $\cdots$ & $\cdots$ \\
\hline 1.5 .1 & 177.39726 & 22.396208 & 18 & 33.1 & $\cdots$ & $\cdots$ \\
\hline 1.5 .2 & 177.39933 & 22.397303 & 18 & 33.2 & $\ldots$ & $\ldots$ \\
\hline 1.5 .3 & 177.40356 & 22.402522 & 18 & 33.3 & $\ldots$ & $\cdots$ \\
\hline 1.6 .1 & 177.39737 & 22.396164 & $\cdots$ & $\ldots$ & 1.1 .13 & $\cdots$ \\
\hline 1.6 .2 & 177.39945 & 22.397236 & $\ldots$ & $\ldots$ & 1.2 .13 & $\cdots$ \\
\hline 1.6 .3 & 177.40360 & 22.402489 & $\ldots$ & $\ldots$ & 1.3 .13 & $\ldots$ \\
\hline 1.7.1 & 177.39757 & 22.396114 & $\ldots$ & 40.1 & $\ldots$ & $\cdots$ \\
\hline 1.7.2 & 177.39974 & 22.396933 & $\ldots$ & 40.2 & $\ldots$ & $\cdots$ \\
\hline 1.7 .3 & 177.40370 & 22.402406 & $\ldots$ & 40.3 & $\ldots$ & $\ldots$ \\
\hline 1.8 .1 & 177.39795 & 22.396014 & $\ldots$ & $\ldots$ & $\cdots$ & $\cdots$ \\
\hline 1.8 .2 & 177.39981 & 22.396750 & $\ldots$ & $\ldots$ & $\ldots$ & $\cdots$ \\
\hline 1.8 .3 & 177.40380 & 22.402311 & $\cdots$ & $\cdots$ & $\cdots$ & $\cdots$ \\
\hline 1.9 .1 & 177.39803 & 22.395939 & $\ldots$ & $\ldots$ & 1.1 .9 & $\cdots$ \\
\hline 1.9 .2 & 177.39973 & 22.396983 & $\ldots$ & $\ldots$ & 1.2 .9 & $\cdots$ \\
\hline 1.9 .3 & 177.40377 & 22.402250 & $\cdots$ & $\cdots$ & 1.3 .9 & $\cdots$ \\
\hline 1.10 .1 & 177.39809 & 22.395856 & $\cdots$ & $\ldots$ & $\cdots$ & $\cdots$ \\
\hline 1.10 .2 & 177.39997 & 22.396708 & $\ldots$ & 36.2 & $\ldots$ & $\cdots$ \\
\hline 1.10 .3 & 177.40380 & 22.402183 & $\cdots$ & 36.3 & $\cdots$ & $\cdots$ \\
\hline 1.11 .2 & 177.40010 & 22.396661 & $\ldots$ & $\ldots$ & 1.2 .3 & $\cdots$ \\
\hline 1.11 .3 & 177.40377 & 22.402047 & $\ldots$ & $\ldots$ & 1.3 .3 & $\ldots$ \\
\hline 1.12 .1 & 177.39716 & 22.395211 & $\ldots$ & $\ldots$ & 1.1 .14 & $\ldots$ \\
\hline 1.12 .2 & 177.40032 & 22.396925 & $\ldots$ & $\ldots$ & 1.2 .14 & $\ldots$ \\
\hline 1.12 .3 & 177.40360 & 22.401878 & $\ldots$ & $\ldots$ & 1.3 .14 & $\cdots$ \\
\hline 1.13 .1 & 177.39697 & 22.396639 & 7 & 24.1 & 1.1 .19 & $\cdots$ \\
\hline 1.13 .2 & 177.39882 & 22.397711 & 7 & 24.2 & 1.2 .19 & $\cdots$ \\
\hline 1.13 .3 & 177.40329 & 22.402828 & 7 & 24.3 & 1.3 .19 & $\cdots$ \\
\hline 1.13 .4 & 177.39791 & 22.398433 & 7 & 24.4 & 1.4 .19 & $\cdots$ \\
\hline 1. ${ }^{*} 13.6$ & 177.39852 & 22.398061 & $\cdots$ & $\ldots$ & $\ldots$ & 3 \\
\hline 1.14 .1 & 177.39712 & 22.396725 & 6 & 25.1 & 1.1 .7 & $\cdots$ \\
\hline 1.14 .2 & 177.39878 & 22.397633 & 6 & 25.2 & 1.2 .7 & $\cdots$ \\
\hline 1.14 .3 & 177.40338 & 22.402872 & 6 & 25.3 & 1.3 .7 & $\cdots$ \\
\hline 1.14 .4 & 177.39810 & 22.398256 & $\ldots$ & 25.4 & 1.4 .7 & $\cdots$ \\
\hline 1.15 .1 & 177.39717 & 22.396506 & $\ldots$ & 41.1 & 1.1 .20 & $\ldots$ \\
\hline 1.15 .2 & 177.39894 & 22.397514 & $\ldots$ & 41.2 & 1.2 .20 & $\ldots$ \\
\hline 1.15 .3 & 177.40344 & 22.402753 & $\ldots$ & 41.3 & 1.3 .20 & $\cdots$ \\
\hline 1.16 .1 & 177.39745 & 22.396400 & 4 & 26.1 & 1.1 .6 & $\cdots$ \\
\hline 1.16 .2 & 177.39915 & 22.397228 & 4 & 26.2 & 1.2 .6 & $\cdots$ \\
\hline 1.16 .3 & 177.40360 & 22.402656 & 4 & 26.3 & 1.3.6 & $\cdots$ \\
\hline 1.17 .1 & 177.39815 & 22.396347 & 3 & 11.1 & 1.1.5 & $\cdots$ \\
\hline 1.17 .2 & 177.39927 & 22.396831 & 3 & 11.2 & 1.2 .5 & $\cdots$ \\
\hline 1.17 .3 & 177.40384 & 22.402564 & 3 & 11.3 & 1.3 .5 & $\ldots$ \\
\hline 1.18 .1 & 177.39850 & 22.396100 & $\cdots$ & $\ldots$ & 1.1 .11 & $\cdots$ \\
\hline 1.18 .2 & 177.39947 & 22.396592 & $\ldots$ & $\ldots$ & 1.2 .11 & $\ldots$ \\
\hline 1.18 .3 & 177.40394 & 22.402408 & $\ldots$ & $\ldots$ & 1.3 .11 & $\ldots$ \\
\hline 1.19 .1 & 177.39689 & 22.395761 & $\ldots$ & 21.1 & 1.1 .17 & $\ldots$ \\
\hline 1.19 .2 & 177.39954 & 22.397486 & $\ldots$ & 21.2 & 1.2 .17 & $\ldots$ \\
\hline
\end{tabular}

Table 4

(Continued)

\begin{tabular}{ccccccc}
\hline \hline 1.19 .3 & 177.40337 & 22.402292 & $\ldots$ & 21.3 & 1.3 .17 & $\ldots$ \\
1.19 .5 & 177.39997 & 22.397106 & $\ldots$ & 21.4 & 1.5 .17 & $\ldots$ \\
1.20 .1 & 177.39708 & 22.395728 & $\ldots$ & 27.1 & 1.1 .16 & $\ldots$ \\
1.20 .2 & 177.39963 & 22.397361 & $\ldots$ & $\ldots$ & 1.2 .16 & $\ldots$ \\
1.20 .3 & 177.40353 & 22.402233 & $\ldots$ & 27.3 & 1.3 .16 & $\ldots$ \\
1.20 .5 & 177.40000 & 22.396981 & $\ldots$ & 27.2 & 1.5 .16 & $\ldots$ \\
1.21 .1 & 177.39694 & 22.395406 & $\ldots$ & $\ldots$ & 1.1 .18 & $\ldots$ \\
1.21 .3 & 177.40341 & 22.402006 & $\ldots$ & $\ldots$ & 1.3 .18 & $\ldots$ \\
1.21 .5 & 177.40018 & 22.397042 & $\ldots$ & $\ldots$ & 1.5 .18 & $\ldots$ \\
1.22 .1 & 177.39677 & 22.395487 & $\ldots$ & $\ldots$ & $\ldots$ & $\ldots$ \\
1.22 .2 & 177.39968 & 22.397495 & $\ldots$ & $\ldots$ & $\ldots$ & $\ldots$ \\
1.22 .3 & 177.40328 & 22.402098 & $\ldots$ & $\ldots$ & $\ldots$ & $\ldots$ \\
1.22 .5 & 177.40008 & 22.397139 & $\ldots$ & $\ldots$ & $\ldots$ & $\ldots$ \\
1.23 .1 & 177.39672 & 22.395381 & 15 & 22.1 & 1.1 .2 & $\ldots$ \\
1.23 .2 & 177.39977 & 22.397497 & 15 & 22.2 & 1.2 .2 & $\ldots$ \\
1.23 .3 & 177.40324 & 22.402011 & 15 & 22.3 & 1.3 .2 & $\ldots$ \\
1.23 .5 & 177.40013 & 22.397200 & $\ldots$ & 22.2 & 1.5 .2 & $\ldots$ \\
1.24 .1 & 177.39650 & 22.395589 & $\ldots$ & 28.1 & 1.1 .4 & $\ldots$ \\
1.24 .2 & 177.39953 & 22.397753 & $\ldots$ & 28.2 & 1.2 .4 & $\ldots$ \\
1.24 .3 & 177.40301 & 22.402203 & $\ldots$ & 28.3 & 1.3 .4 & $\ldots$ \\
1.25 .1 & 177.39657 & 22.395933 & $\ldots$ & $\ldots$ & 1.1 .21 & $\ldots$ \\
1.25 .3 & 177.40304 & 22.402456 & $\ldots$ & $\ldots$ & 1.3 .21 & $\ldots$ \\
1.27 .1 & 177.39831 & 22.396285 & $\ldots$ & 37.1 & $\ldots$ & $\ldots$ \\
1.27 .2 & 177.39933 & 22.396725 & $\ldots$ & 37.2 & $\ldots$ & $\ldots$ \\
1.26 .1 & 177.39633 & 22.396011 & $\ldots$ & $\ldots$ & 1.1 .12 & $\ldots$ \\
1.26 .3 & 177.40283 & 22.402600 & $\ldots$ & $\ldots$ & 1.3 .12 & $\ldots$ \\
1.28 .1 & 177.39860 & 22.396166 & $\ldots$ & 38.1 & $\ldots$ & $\ldots$ \\
1.28 .2 & 177.39942 & 22.396559 & $\ldots$ & 38.2 & $\ldots$ & $\ldots$ \\
1.29 .1 & 177.39858 & 22.395860 & $\ldots$ & 39.1 & $\ldots$ & $\ldots$ \\
1.29 .2 & 177.39976 & 22.396490 & $\ldots$ & 39.2 & $\ldots$ & $\ldots$ \\
1.30 .1 & 177.39817 & 22.395465 & $\ldots$ & 35.1 & $\ldots$ & $\ldots$ \\
1.30 .2 & 177.39801 & 22.395230 & $\ldots$ & 35.2 & $\ldots$ & $\ldots$ \\
1.30 .3 & 177.39730 & 22.395364 & $\ldots$ & 35.3 & $\ldots$ & $\ldots$ \\
1.30 .4 & 177.39788 & 22.395721 & $\ldots$ & 35.4 & $\ldots$ & $\ldots$ \\
$\mathrm{SN} 1$ & 177.39823 & 22.395631 & $\ldots$ & 30.1 & $1.1 .3 \mathrm{a}$ & $\ldots$ \\
$\mathrm{SN} 2$ & 177.39772 & 22.395783 & $\ldots$ & 30.2 & $1.1 .3 \mathrm{~b}$ & $\ldots$ \\
$\mathrm{SN} 3$ & 177.39737 & 22.395539 & $\ldots$ & 30.3 & $1.1 .3 \mathrm{c}$ & $\ldots$ \\
$\mathrm{SN} 4$ & 177.39781 & 22.395189 & $\ldots$ & 30.4 & $1.1 .3 \mathrm{~d}$ & $\ldots$ \\
\hline & & $\ldots$ \\
Nes & $\ldots$ & $\ldots$ & \\
& & $\ldots$ & $\ldots$ \\
\end{tabular}

Notes. Coordinates and ID notations of emission knots in the multiply imaged host of SN Refsdal, at $z=1.489$. The labels in previous publications are indicated. New identifications were made by C.G., K.S., and J.D. Each modeling team used a modified version or subset of the list above, with the coordinates of each knot varying slightly between modelers. Nevertheless, there is consensus among the modelers on the identification and mapping of the different features between the multiple images of the same source. (1) Images 1.1, 1.2, 1.3, and 1.5 were labeled by Zitrin \& Broadhurst (2009) as 1.2, 1.3, 1.1 , and 1.4, respectively. The labels of other knots were not given in that publication. (2) This knot was identified as a counterimage of the bulge of the galaxy by Zitrin \& Broadhurst (2009), but rejected by Smith et al. (2009). As in the paper by Sharon \& Johnson (2015), the modelers' consensus is that this knot is likely at least a partial image of the bulge. (3) Image 1.13.6 is predicted by some models to be a counterimage of 1.13 , but its identification is not sufficiently confident to be used as constraint.

(Bradač et al. 2004b, 2009). However, given the pixelated nature of the reconstruction and the need to compute numerical derivatives and interpolate from noisy pixels in order to compute time delays and magnifications at the location of SN Refsdal, we did not expect this method to be competitive for this specific application. Therefore, in the interest of time, we did not construct this model. A pre-HFF model of MACSJ1149.5+2223 using this approach is available through the HFF website and will be updated in the future. 

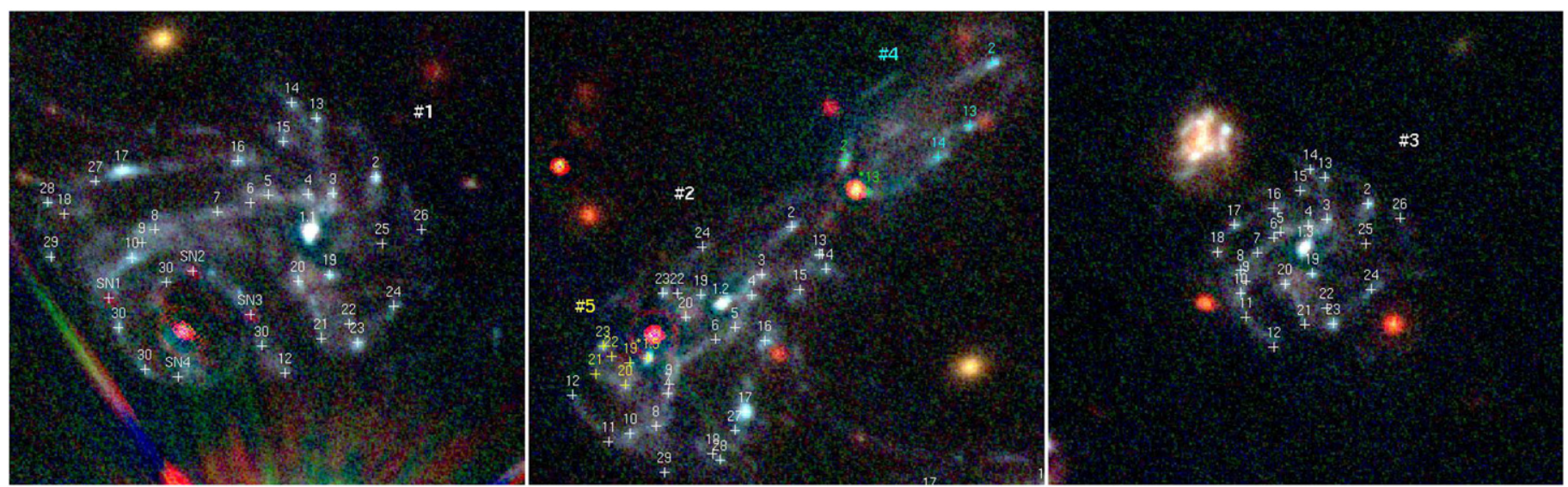

Figure 3. Knots and morphological features in the host galaxy of SN Refsdal at $z=1.489$. The color composite on which the regions are overplotted is generated by scaling and subtracting the F814W image from the F435W, F606W, and F105W images, in order to suppress the light from the foreground cluster galaxies. The left panel shows image 1.1, and the right panel shows image 1.3. In the middle panel, the complex lensing potential in the central region is responsible for one full image, 1.2, and additional partial images of the galaxy, 1.4, and 1.5 (see also Smith et al. 2009; Zitrin et al. 2009, and Sharon \& Johnson 2015). To guide the eye, we label knots that belong to 1.4 and 1.5 in cyan and yellow, respectively. A possible sixth image of a small region of the galaxy is labeled in green. The two features marked with an asterisk in this panel, ${ }^{*} 1.5$ and ${ }^{*} 13$, are the only controversial identifications. We could not rule out the identification of *1.5 (knot 1.1 .5 in Table 4 ) as a counterpart of the bulge of the galaxy; however, it is likely only partly imaged. Image *13 (1.13.6 in Table 4) is suggested by some of the models, but hard to confirm, and is thus not used as a constraint in the gold lens models considered here. We note that the exact coordinates of each feature may vary slightly between modelers, and we refer the reader to detailed publications (in preparation) by each modeling team for exact positions and features used.

Table 5

Summary of Models

\begin{tabular}{lcccc}
\hline \hline Short name & Team & Type & rms & Images \\
\hline Die-a & Diego et al. & Free-form & 0.78 & gold + sil \\
Gri-g & Grillo et al. & Simply param & 0.26 & gold \\
Ogu-g & Oguri et al. & Simply param & 0.43 & gold \\
Ogu-a & Oguri et al. & Simply param & 0.31 & all \\
Sha-g & Sharon et al. & Simply param & 0.16 & gold \\
Sha-a & Sharon et al. & Simply param & 0.19 & gold + sil \\
Zit-g & Zitrin et al. & Light-tr-mass & 1.3 & gold \\
\hline
\end{tabular}

Note. For each model we provide a short name as well as basic features and inputs. The column rms lists the root mean square scatter of the observed versus predicted image positions in arcseconds.

When appropriate, we also describe additional sets of constraints used by each modeler.

\subsection{Diego et al.}

A full description of the modeling technique used by this team (J. D., T. B.) and the various improvements implemented in the code can be found in the literature (Diego et al. 2005, 2007, 2015; Sendra et al. 2014). Here is a brief summary of the basic steps.

\subsubsection{Definition of the Mass Model}

The algorithm (WSLAP+) relies on a division of the mass distribution in the lens plane into two components. The first is compact and associated with the member galaxies (mostly red ellipticals). The second is diffuse and distributed as a superposition of Gaussians on a regular (or adaptive) grid. In this specific case, a grid of $512 \times 512$ pixels 0 "' 1875 on a side was used. For the compact component, the mass associated with the galaxies is assumed to be proportional to their luminosity. If all the galaxies are assumed to have the same mass-to-light $(M / L)$ ratio, the compact component (galaxies) contributes with just one $\left(N_{g}=1\right)$ extra free parameter which corresponds to the correction that needs to be applied to the fiducial $M / L$ ratio. In a few particular cases, some galaxies (like the brightest cluster galaxy (BCG) or massive galaxies very close to an arclet) are allowed to have their own $M / L$ ratio, adding additional free parameters to the lens model but typically no more than a few $\left(N_{g} \approx \mathrm{O}(1)\right)$. For this component associated with the galaxies, the total mass is assumed to follow either a (Navarro et al. 1997, hereafter NFW) profile (with a fixed concentration, and scale radius scaling with the fiducial halo mass) or be proportional to the observed surface brightness. For this work the team adopted $N_{g}=2$ or $N_{g}=3$. The case $N_{g}=2$ considers one central BCG and the elliptical galaxy near image 1.2 to have the same $M / L$ ratio, while the remaining galaxies have a different one. In the case $N_{g}=3$, the BCG and the galaxy near image 1.2 each have their own $M / L$ ratio, and the remaining galaxies are assumed to have a third independent value. In all cases, it is important to emphasize that the member galaxy between the 4 observed images of SN Refsdal was not allowed to have its own independent $M / L$ ratio. This results in a model that is not as accurate on the smallest scales around this galaxy as other models that allow this galaxy to vary.

The diffuse or "soft" component is described by as many free parameters as grid (or cell) points. This number $\left(N_{c}\right)$ varies but is typically between a few hundred to one thousand $\left(N_{c} \approx\right.$ $\mathrm{O}(100)-\mathrm{O}(1000)$ ) depending on the resolution and/or use of the adaptive grid. In addition to the free parameters describing the lens model, the problem includes as unknowns the original positions of the lensed galaxies in the source plane. For the clusters included in the HFF program the number of background sources, $N_{\mathrm{s}}$, is typically a few tens $\left(N_{\mathrm{s}} \approx \mathrm{O}(10)\right)$, each contributing with two unknowns $\left(\beta_{x}\right.$ and $\left.\beta_{y}\right)$. All of the unknowns are then combined into a single array $X$ with $N_{x}$ elements $\left(N_{x} \approx O(1000)\right)$.

\subsubsection{Definition of the Inputs}

The inputs are the pixel position of the strongly lensed galaxies (not just the centroids) for all the multiple images 
listed in Tables 3 and 4 . In the case of elongated arcs near the critical curves with no features, the entire arc is mapped and included as a constraint. If the arclets have individual features, these can be incorporated as semi-independent constraints but with the added condition that they need to form the same source in the source plane. The following inputs are added to the default set of image and knot centers listed in Section 3.

1. Shape of the arclets. This is particularly useful for long elongated arcs (with no counterimages) which lie in the regime between the weak and strong lensing. These arcs are still useful constraints that add valuable information beyond the Einstein radius.

2. Shape and morphology of arcs. By including this information one can account (at least partially) for the magnification at a given position.

3. Resolved features in individual systems. This new addition to the code is motivated by the host galaxy of SN Refsdal, where multiple features can be identified in the different counter images. In addition, the counterimage in the north, when relensed, offers a robust picture of the original source morphology (size, shape, orientation). This information acts as an anchor, constraining the range of possible solutions.

Weak lensing shear measurements can also be used as input to the inference. For the particular case of MACSJ1149.5 +2223 the weak lensing measurements are not used, to ensure homogeneity with the other methods.

\subsubsection{Description of the Inference Process and Error Estimation}

The array of best-fit parameters, $X$, is obtained after solving the system of linear equations

$$
\Theta=\Gamma X
$$

where the $N_{o}$ observations (strong lensing, weak lensing, time delays) are included in the array $\Theta$, and the matrix $\Gamma$ is known and has dimension $N_{o} \times\left(N_{c}+N_{g}+2 N_{\mathrm{s}}\right)$.

In practice, $X$ is obtained by solving the set of linear equations described in Equation (1) via a fast biconjugate algorithm, or inverted with a singular value decomposition (after setting a threshold for the eigenvalues) or solved with a more robust but slower quadratic algorithm. The quadratic algorithm is the preferred method, as it imposes the physical constraint that the solution $X$ must be positive. This eliminates unphysical solutions with negative masses and reduces the space of possible solutions. Like in the case of the biconjugate gradient, the quadratic programming algorithm solves the system of linear equations by finding the minimum of the associated quadratic function. Errors in the solution are derived by minimizing the quadratic function multiple times, after varying the initial conditions of the minimization process, and/ or varying the grid configuration.

\subsection{Grillo et al.}

The software used by this team (C. G., S. H. S., A. H., P. R., W. K., I. B., A. M., G. B. C.) is GleE (Suyu \& Halkola 2010; Suyu et al. 2012). The strong lensing analysis performed here follows very closely the one presented by Grillo et al. (2015b) for another HFF target, MACSJ0416.1-2403. Cosmological applications of GLEE can be found in the papers by Suyu et al. (2013, 2014), and further details on the strong lensing modeling of MACSJ1149.5+2223 are provided in a dedicated paper (Grillo et al. 2015a).

\subsubsection{Definition of the Mass Model}

Different mass models have been explored for this galaxy cluster, but only the best-fitting one is discussed here. The projected dimensionless total surface mass density of 300 cluster members within the WFC3 field of view of the CLASH observations is modeled as a dual pseudoisothermal elliptical mass distribution (dPIE; Elíasdóttir et al. 2007) with vanishing ellipticity and core radius. The zero-core dPIE profile corresponds to the three-dimensional mass density profile:

$$
\rho(r) \propto \frac{1}{r^{2}\left(r^{2}+r_{t}^{2}\right)} .
$$

The galaxy luminosity values in the F160W band are used to assign the relative weights to their total mass profile. The galaxy total $M / L$ ratio is scaled with luminosity as $M_{\mathrm{T}} / L \propto L^{0.2}$, thus mimicking the so-called "tilt" of the Fundamental Plane. The values of axis ratio, PA, effective velocity dispersion, and truncation radius of the two cluster members closest in projection to the central and southern images of the SN Refsdal host are left free. To complete the total mass distribution of the galaxy cluster, three additional mass components are added to describe the cluster dark matter halo on physical scales larger than those typical of the individual cluster members. These cluster halo components are parametrized as two-dimensional pseudo-isothermal elliptical mass profiles (PIEMD as defined by Kassiola \& Kovner 1993; see also Grillo et al. 2015a).

No external shear or higher-order perturbations are included in the model. The number of free parameters associated with the model of the cluster total mass distribution is 28 .

\subsubsection{Definition of the Inputs}

The positions of the multiple images belonging to the 10 systems of the gold sample and to 18 knots of the SN Refsdal host are the observables over which the values of the model parameters are optimized. The adopted positional uncertainty of each image is 0 ! 065 . The redshift values of the 7 spectroscopically confirmed gold systems are fixed, while the remaining 3 systems are included with a uniform prior on the value of $D_{\mathrm{ds}} / D_{\mathrm{s}}$, where $D_{\mathrm{ds}}$ and $D_{\mathrm{s}}$ are the deflector-source and observer-source angular diameter distances, respectively. In total, 88 observed image positions are used to reconstruct the cluster total mass distribution.

\subsubsection{Description of the Inference Process and Error Estimation}

The best-fitting, minimum- $\chi^{2}$ model is obtained by minimizing the distance between the observed and model-predicted positions of the multiple images in the lens plane. A minimum $\chi^{2}$ value of 1441 , corresponding to an rms offset between the image observed and reconstructed positions of 0.26 , is found. To sample the posterior probability distribution function of the model parameters, the image positional uncertainty is increased until the value of the $\chi^{2}$ is comparable to the number of the degrees of freedom (89), and standard Markov chain Monte Carlo (MCMC) methods are used. The quantities shown in Figures 9 to 12 are for the model-predicted images of SN 
Refsdal and are obtained from 100 different models extracted from an MCMC chain with $10^{6}$ samples and an acceptance rate of approximately 0.13 .

\subsection{Oguri et al.}

\subsubsection{Definition of the Mass Model}

This team (M. O., M. I., R. K.) uses the public software GLAFIC (Oguri 2010). This "simply parametrized" method assumes that the lens potential consists of a small number of components describing dark halos, cluster member galaxies, and perturbations in the lens potential. The dark halo components are assumed to follow the elliptical NFW mass density profile. In contrast, the elliptical pseudo-Jaffe profile is adopted to describe the mass distribution of cluster member galaxies. In order to reduce the number of free parameters, the velocity dispersion $\sigma$ and the truncation radius $r_{\text {cut }}$ for each galaxy are assumed to scale with the ( $F 814 W$-band) luminosity of the galaxy as $\sigma \propto L^{1 / 4}$ and $r_{\text {cut }} \propto L^{\eta}$, with $\eta$ being a free parameter. In addition, the second-order (external shear) and third-order perturbations are included so as to account for asymmetry of the overall lens potential. Interested readers are referred to Oguri $(2010,2015)$, Oguri et al. (2012, 2013), and Ishigaki et al. (2015) for more detailed descriptions and examples of cluster mass modeling with GLAFIC. Additional details are given in a dedicated paper (Kawamata et al. 2015).

\subsubsection{Definition of the Inputs}

The positions of multiple images and knots listed in Section 3 are used as constraints. Image 1.5 was not used as a constraint. To accurately recover the position of SN Refsdal, different positional uncertainties are assumed for different multiple images. Specifically, while the positional uncertainty of $0 . " 4$ in the image plane is assumed for most of the multiple images, smaller positional uncertainties of $0 . \prime 05$ and $0 . " 2$ are assumed for SN Refsdal and knots of the SN host galaxy, respectively (see also Oguri 2015). When spectroscopic redshifts are available, their redshifts are fixed to the spectroscopic redshifts. Otherwise source redshifts are treated as model parameters and are optimized simultaneously with the other model parameters. For a subsample of multiple image systems for which photometric redshift estimates are secure and accurate, a conservative Gaussian prior with a dispersion of $\sigma_{z}=0.5$ for the source redshift is added. While GLAFIC allows one to include other types of observational constraints, such as flux ratios, time delays, and weak lensing shear measurements, those constraints are not used in the mass modeling of MACSJ1149.5+2223.

\subsubsection{Description of the Inference Process and Error Estimation}

The best-fit model is obtained simply by minimizing $\chi^{2}$. The so-called source plane $\chi^{2}$ minimization is used for an efficient model optimization (see Appendix 2 of Oguri 2010). A standard MCMC approach is used to estimate errors on model parameters and their covariance.

The predicted time delays and magnifications are computed at the model-predicted positions. For each mass model (chain), the best-fit source position of the $\mathrm{SN}$ is derived. From that, the corresponding SN image positions in the image plane (which can be slightly different from observed $\mathrm{SN}$ positions) are obtained for that model, and finally the time delays and magnifications of the images are calculated.

\subsection{Sharon et al.}

The approach of this team (K. S., T. J.) was based on the publicly available software Lenstool (Jullo et al. 2007). Lenstool is a "simply parametrized" lens modeling code. In practice, the code assumes that the mass distribution of the lens can be described by a combination of mass halos, each of them taking a functional form whose properties are defined by a set of parameters. The method assumes that mass generally follows light, and assigns halos to individual galaxies that are identified as cluster members. Cluster- or group-scale halos represent the cluster mass components that are not directly related to galaxies. The number of cluster or group-scale halos is determined by the modeler. Typically, the positions of the cluster-scale halos are not fixed and are left to be determined by the modeling algorithms. A hybrid "simply parametrized"/ "free-form" approach has also been implemented in Lenstool (Jullo \& Kneib 2009), where numerous halos are placed on a grid, representing the overall cluster component. This hybrid method is not implemented in this work.

\subsubsection{Definition of the Mass Model}

The halos are represented by elliptical mass distributions corresponding to a spherical density profile $\rho(r)$ described by the equation

$$
\rho(r)=\frac{\rho_{0}}{\left(1+r^{2} / r_{\text {core }}^{2}\right)\left(1+r^{2} / r_{\text {cut }}^{2}\right)} .
$$

These halos are isothermal at intermediate radii, i.e., $\rho \propto r^{-2}$ at $r_{\text {core }} \lesssim r \lesssim r_{\text {cut }}$, and they have a flat core internal to $r_{\text {core }}$. The profile is equivalent to that given in Equation (2) for $r_{\text {core }}=0$. It is sometimes known as dPIE or "truncated PIEMD" (Elíasdóttir et al. 2007), although it differs from the original PIEMD profiled defined by Kassiola \& Kovner (1993). The transition between the different slopes is smooth. The quantity $\sigma_{0}$ defines the overall normalization as a fiducial velocity dispersion. In Lenstool, each of these halos has seven free parameters: centroid position $(x, y)$; ellipticity $e=\left(a^{2}-b^{2}\right) /\left(a^{2}+b^{2}\right)$ where $a$ and $b$ are the semimajor and semiminor axes, respectively; PA $\theta$; and $r_{\text {core }}, r_{\text {cut }}$, and $\sigma_{0}$ as defined above.

The selection of cluster member galaxies is described in Section 3.3. In this model, 286 galaxies were selected from the cluster member catalog, by a combination of their luminosity and projected distance from the cluster center, such that the deflection caused by an omitted galaxy is much smaller than the typical uncertainty caused by unseen structure along the line of sight. This selection criterion results in removal of faint galaxies at the outskirts of the cluster, and inclusion of all the galaxies that pass the cluster-member selection in the core.

Cluster member galaxies are also modeled with the profile given by Equation (3). Their positional parameters are fixed on their observed properties as measured with SExtractor (Bertin $\&$ Arnouts 1996) for $x, y, e$, and $\theta$. The other parameters- $r_{\text {core }}$, $r_{\text {cut }}$, and $\sigma_{0}$-are linked to their luminosity in the $F 814 \mathrm{~W}$ band through scaling relations (e.g., Limousin et al. 2005) assuming 
a constant $M / L$ ratio for all galaxies,

$$
\sigma_{0}=\sigma_{0}^{*}\left(\frac{L}{L^{*}}\right)^{1 / 4} \text { and } r_{\text {cut }}=r_{\text {cut }}^{*}\left(\frac{L}{L^{*}}\right)^{1 / 2} .
$$

\subsubsection{Definition of the Inputs}

The lensing constraints are the positions of multiple images of each lensed source, plus those of the knots in the host galaxy of SN Refsdal, as listed in Section 3. In cases where the lensed image is extended or has substructure, the exact positions were selected to match similar features within multiple images of the same galaxy with each other, thus obtaining more constraints, a better local sampling of the lensing potential, and a better handle on the local magnification. Where available, spectroscopic redshifts are used as fixed constraints. For sources with no spectroscopic redshift, the redshifts are considered as free parameters with photometric redshifts informing their Bayesian priors. The uncertainties of the photometric redshifts are relaxed in order to allow for outliers (to an interval of approximately $\delta z= \pm 2$ around the photo- $z$ ). We present two models here: Sha-g uses as constraints the gold sample of multiply imaged galaxies, and Sha-a uses gold, silver, and secure arcs outside the MUSE field of view, to allow better coverage of lensing evidence in the outskirts of the cluster and, in particular, to constrain the subhalos around MACSJ1149.5+2223.

\subsubsection{Description of the Inference Process and Error Estimation}

The parameters of each halo are allowed to vary under Bayesian priors, and the parameter space is explored in an MCMC process to identify the set of parameters that provide the best fit. The quality of the lens model is measured either in the source plane or in the image plane. The latter requires significantly longer computation time. In source-plane minimization, the source positions of all the images of each set are computed by ray tracing the image-plane positions through the lens model to the source plane. The best-fit model is the one that results in the smallest scatter in the source positions of multiple images of the same source. In image-plane minimization, the model-predicted counterimages of each of the multiple images of the same source are computed. This results in a set of predicted images near the observed positions. The best-fit model is the one that minimizes the scatter among these imageplane positions. The MCMC sampling of the parameter space is used to estimate the statistical uncertainties that are inherent to the modeling algorithm. In order to estimate the uncertainties on the magnification and time delay, potential maps are generated from sets of parameters from the MCMC chain that represent $1 \sigma$ in the parameter space.

\subsection{Zitrin et al}

\subsubsection{Definition of the Mass Model}

The method used by this team (A. Z.) is a Light Traces Mass (LTM) method, so that both the galaxies and the dark matter follow the light distribution. The method is described in detail by Zitrin et al. (2009, 2013), and it is inspired by the LTM assumptions outlined by Broadhurst et al. (2005). The model consists of two main components. The first component is a mass map of the cluster galaxies, chosen by following the red sequence. Each galaxy is represented with a power-law surface mass density distribution, where the surface density is proportional to its surface brightness. The power law is a free parameter of the model and is iterated (all galaxies are forced to have the same exponent). The second component is a smooth dark matter map, obtained by smoothing (with a spline polynomial or with a Gaussian kernel) the first component (i.e., the superposed red sequence galaxy mass distribution). The smoothing degree is the second free parameter of the model. The two components are then added with a relative weight which is a free parameter, along with the overall normalization.

Next, a two-component external shear can be included to add flexibility and generate ellipticity in the magnification map. Finally, individual galaxies can be assigned with free masses to be optimized by the minimization procedure, to allow more degrees of freedom deviating from the initial imposed LTM. This procedure has been shown to be very effective in locating multiple images in many clusters (e.g., Zitrin et al. 2009, 2012b, 2013, 2015), even without any multiple images initially used as input (Zitrin et al. 2012a). Most of the multiple images in MACSJ1149.5+2223 that were found by Zitrin \& Broadhurst (2009) and Zheng et al. (2012) were identified with this method.

\subsubsection{Definition of the Inputs}

All sets of multiple images in the gold list were used except system 14. Most knots were used except those in the fifth radial BCG image. All systems listed with spec- $z$ (aside for system 5) were kept fixed at that redshift, while all other gold systems were left to be freely optimized with a uniform flat prior. Image position uncertainties were adopted to be $0 . " 5$, aside for the four SN images for which 0 ." 15 was used.

\subsubsection{Description of the Inference Process and Error Estimation}

The best-fit solution and uncertainties are obtained via converged MCMC chains.

\section{COMPARISON OF LENS MODELS}

In this section we carry out a comparison of the 7 models, focusing specifically on the quantities that are relevant for SN Refsdal. We start in Section 5.1 by presenting the twodimensional maps of convergence, magnification, and time delay, for a deflector at the redshift of the cluster and a source at the redshift of SN Refsdal $(z=1.489$; we note that assuming $z=1.491$, the redshift published by Smith et al. (2009), would not have made any significant difference). Then, in Section 5.2, we compare quantitatively the predicted time delays and magnification ratios of the known images with their measured values. Finally, in Section 5.3 we present the forecast for the future (and past) SN images. All of the lens models predict the appearance of an image of the $\mathrm{SN}$ in the two other images of the host galaxy. In the following sections, we refer to the predicted SN in image 1.2 of the host galaxy as SX, and the one in image 1.3 of the host as SY, following the labeling of previous publications. The predicted time delays and magnification ratios are given in Table 6.

\subsection{Convergence, Magnification, and Time-Delay Maps}

Figure 4 shows the convergence (i.e., surface mass density in units of the lensing critical density) maps. There are striking qualitative differences. The Zit-g map is significantly rounder 
Table 6

Summary of Predicted Time Delays and Magnification Ratios

\begin{tabular}{|c|c|c|c|c|c|c|c|c|c|c|}
\hline Model & $\Delta t_{21}$ & $\Delta t_{31}$ & $\Delta t_{41}$ & $\Delta t_{\mathrm{X} 1}$ & $\Delta t_{\mathrm{Y} 1}$ & $\mu(2) / \mu(1)$ & $\mu(3) / \mu(1)$ & $\mu(4) / \mu(1)$ & $\mu(X) / \mu(1)$ & $\mu(Y) / \mu(1)$ \\
\hline Die-a & $-17 \pm 19$ & $-4.0 \pm 27$ & $74 \pm 43$ & $262 \pm 55$ & $-4521 \pm 524$ & $1.89 \pm 0.79$ & $0.64 \pm 0.19$ & $0.35 \pm 0.11$ & $0.31 \pm 0.10$ & $0.41 \pm 0.11$ \\
\hline Gri-g & $10.6_{-3.0}^{+6.2}$ & $4.8_{-1.8}^{+3.2}$ & $25.9_{-4.3}^{+8.1}$ & $361_{-27}^{+19}$ & $-6183_{-145}^{+160}$ & $0.92_{-0.52}^{+0.43}$ & $0.99_{-0.33}^{+0.52}$ & $0.42_{-0.20}^{+0.19}$ & $0.36_{-0.09}^{+0.11}$ & $0.30_{-0.07}^{+0.09}$ \\
\hline Ogu-g & $8.7 \pm 0.7$ & $5.1 \pm 0.5$ & $18.8 \pm 1.7$ & $311 \pm 24$ & $-5982 \pm 287$ & $1.14 \pm 0.24$ & $1.22 \pm 0.24$ & $0.67 \pm 0.17$ & $0.27 \pm 0.05$ & $0.19 \pm 0.03$ \\
\hline Ogu-a & $9.4 \pm 1.1$ & $5.6 \pm 0.5$ & $20.9 \pm 2.0$ & $336 \pm 21$ & $-6239 \pm 224$ & $1.15 \pm 0.17$ & $1.19 \pm 0.17$ & $0.64 \pm 0.11$ & $0.27 \pm 0.03$ & $0.23 \pm 0.03$ \\
\hline Sha-g & $6_{-5}^{+6}$ & $-1_{-5}^{+7}$ & $12_{-3}^{+3}$ & $277_{-21}^{+11}$ & $-5016_{-15}^{+281}$ & $0.84_{-0.06}^{+0.18}$ & $1.68_{-0.21}^{+0.55}$ & $0.57_{-0.04}^{+0.11}$ & $0.25_{-0.02}^{+0.05}$ & $0.19_{-0.01}^{+0.03}$ \\
\hline Sha-a & $8_{-5}^{+7}$ & $5_{-7}^{+10}$ & $17_{-5}^{+6}$ & $233_{-13}^{+46}$ & $-4860_{-305}^{+126}$ & $0.84_{-0.19}^{+0.20}$ & $1.46_{-0.49}^{+0.07}$ & $0.44_{-0.10}^{+0.05}$ & $0.19_{-0.04}^{+0.01}$ & $0.17_{-0.03}^{+0.01}$ \\
\hline Zit-g & $-161 \pm 97$ & $-149 \pm 113$ & $82 \pm 51$ & $224 \pm 262$ & $-7665 \pm 730$ & $6.27 \pm 0.41$ & $0.83 \pm 0.05$ & $3.69 \pm 0.45$ & $0.31 \pm 0.05$ & $0.30 \pm 0.02$ \\
\hline
\end{tabular}

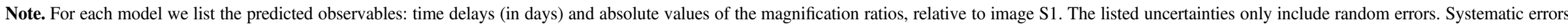
are nonnegligible and described in detail in Section 6.1. 

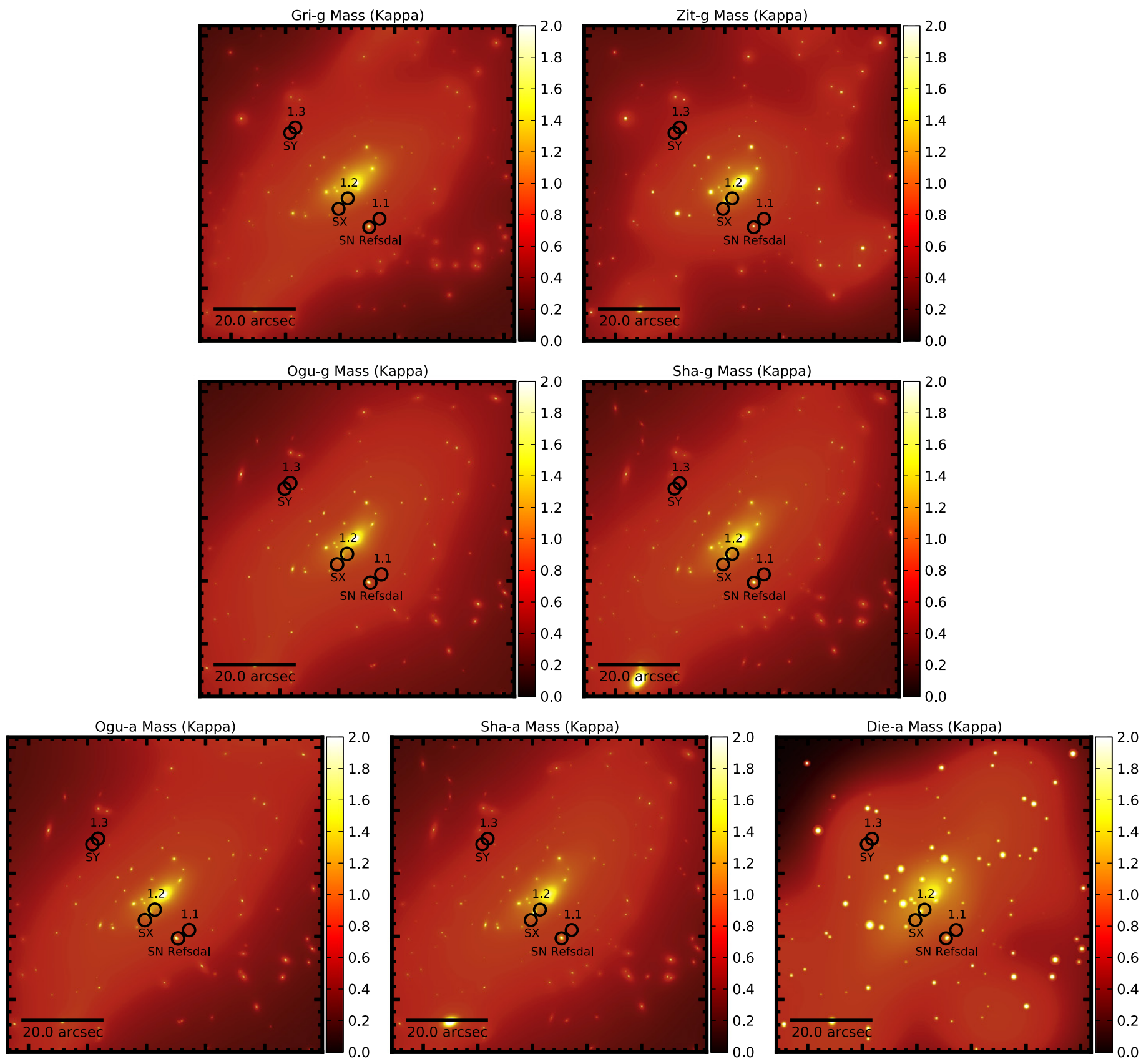

Figure 4. Comparing the mass distributions for the models, labeled as in Table 4. Convergence is computed relative to the critical density with the deflector at the redshift of the cluster and the source at the redshift of the SN. The circles identify the positions of the observed and predicted images of SN Refsdal and those of the multiple images of its host galaxy. The top four panels are models including only the gold sample of images as constraints.

than the others. The Die-a map has significantly more structure, notably two overdensities near SY/1.3 and at the bottom right of the map. These features were to be expected based on the assumptions used by their methods. The Grillo, Oguri, and Sharon convergence maps are the most qualitatively similar. This is perhaps unsurprising since the three codes are based on fairly similar assumptions.

Magnification maps are shown in Figure 5. The regions of extreme magnification are qualitatively similar, even though, similarly to the convergence maps, the Zit-g model is overall rounder, while the Die-a model has more structure.

The time-delay surfaces are illustrated at three zoom levels to highlight different features. Figure 6 shows the global topology of the time-delay surfaces, which is very similar for all models, with minima near 1.1 and $\mathrm{SY} / 1.3$ and a saddle point near $\mathrm{SX} / 1.2$. As was the case with convergence and magnification, the Zit-g and Die-a time-delay surfaces are rounder and have more structure, respectively, than those produced by the other models.

Zooming in on the region of SX/1.2 and 1.1 in Figure 7 reveals more differences. The locations of the minimum near SN Refsdal and of the saddle point near 1.2 are significantly different for the Zit-g model, seemingly as a result of the different contribution of the bright galaxy to the NW of 1.2.

A further zoom-in on the region of the known images is shown in Figure 8. The time-delay surface contour levels are shown in step of 10 days to highlight the behavior relevant for the cross configuration. Whereas the "simply parametrized" 

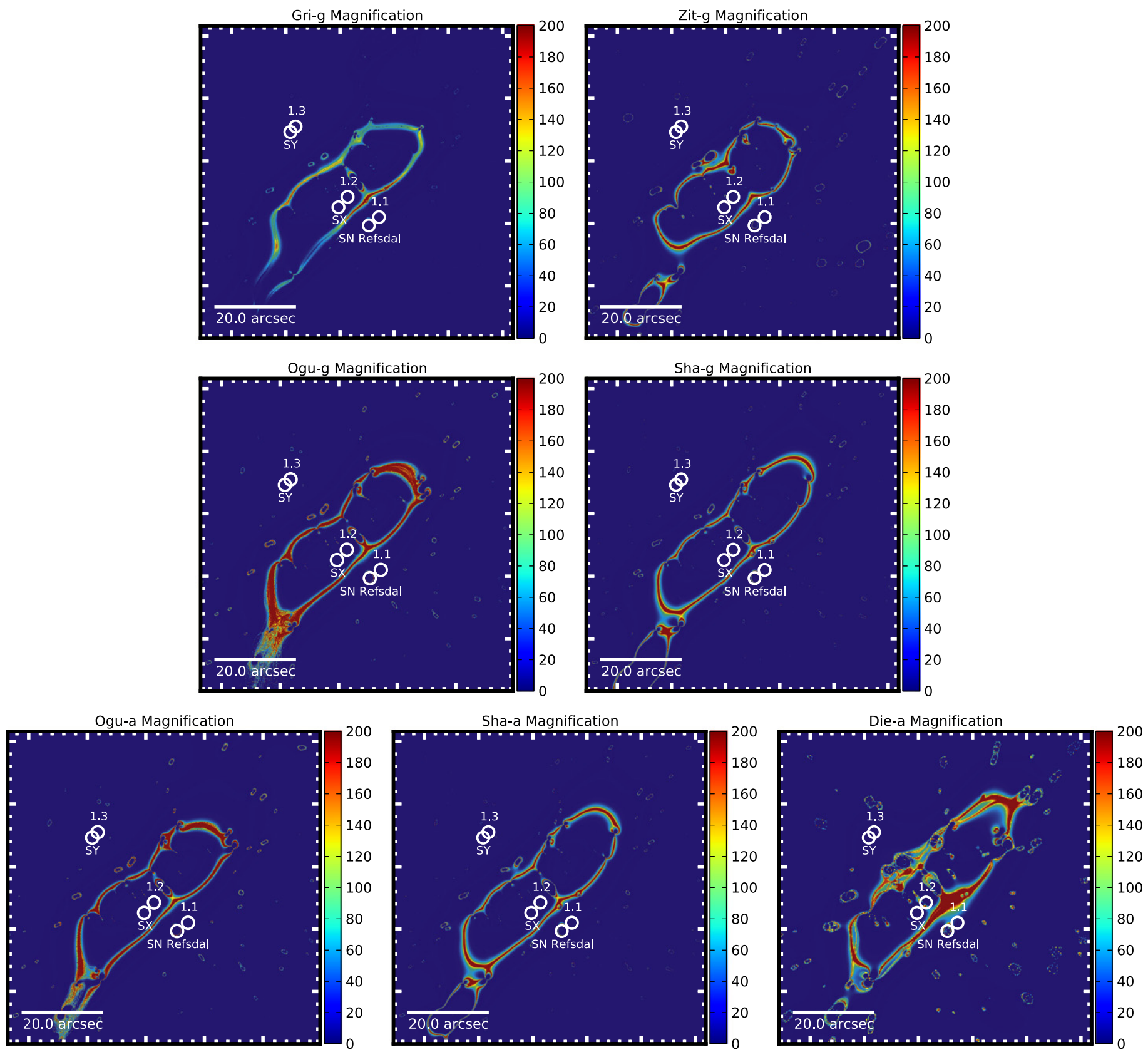

Figure 5. As in Figure 4 for magnification.

models are topologically very similar to each other, the Die-a and Zit-g models are qualitatively different. The time-delay surface is shifted upward, probably as a result of the nearby perturber highlighted in the previous paragraph. We stress that all of the models here are global models, developed to reproduce the cluster potential on larger scales. Hence, local differences should be expected, even though of course they are particularly important in this case.

\subsection{Comparing Model Predictions with Measured Time Delays and Magnification Ratios}

Before proceeding with a quantitative comparison, we emphasize once again that the uncertainties discussed in this section include only statistical uncertainties. Furthermore, in the comparison we neglect for computational reasons the covariance between the predictions for each of the images, both in time delays and in magnification. Systematic uncertainties will be discussed in Section 6 .

Figure 9 compares the measured time delays with those predicted by the models for the cross configuration. We stress that the measurements were not used in the construction of the models (or known to the modelers), and therefore they can be considered an independent test of the models. The time delay between S2 and S1 (and to some extent that between S3 and $\mathrm{S} 1$ ) is very short, and in fact not all the models agree on the ordering of the two images. The time delay between S4 and S1 is longer and better behaved, with all the models agreeing on the order of the images and with the measured value within the uncertainties. Overall, the models are in reasonable agreement with the measurements, even though formally some of them are in statistical tension. This tension indicates that the uncertainties for some of the parametric models are underestimated. 

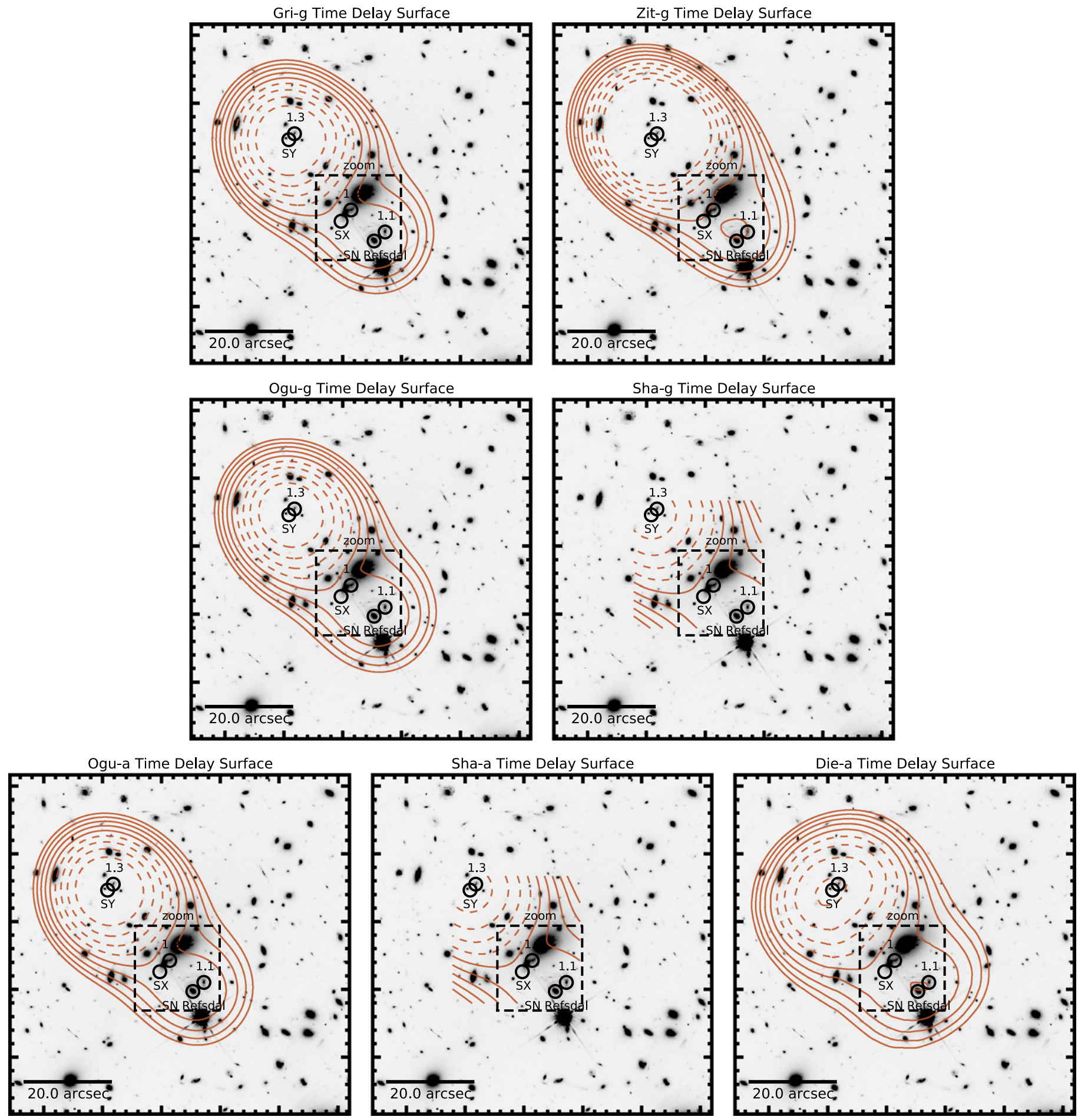

Figure 6. As in Figure 4 for time-delay surfaces. The dashed boxes mark the location of the zoom-in regions shown in Figure 7. Contour levels indicate the time delay from -12 to 12 years in increments of 3 years, relative to S1. For the Sha-a and Sha-g models the time-delay surfaces were only calculated in the region shown. Negative levels are marked by dashed contours. The gray-scale background image shows the HFF F140W epoch2 version 1.0 mosaic.

Interestingly, the models appear to predict rather accurately the observed magnification ratios (Figure 10), even though these quantities should be more sensitive to systematic uncertainties arising from millilensing and microlensing effects than time delays.

Overall, the Zit-g model stands apart from the rest, predicting significantly different time delays and magnification ratios, and larger uncertainties. This qualitative difference is consistent with the different topology of the time-delay surface highlighted in the previous section. Quantitatively, however, the Zit-g model predictions are in broad agreement with the measurements if one considers the $95 \%$ credible interval. Collectively, the "simply parametrized" models seem to predict smaller uncertainties than the others, especially the Ogu-g and 

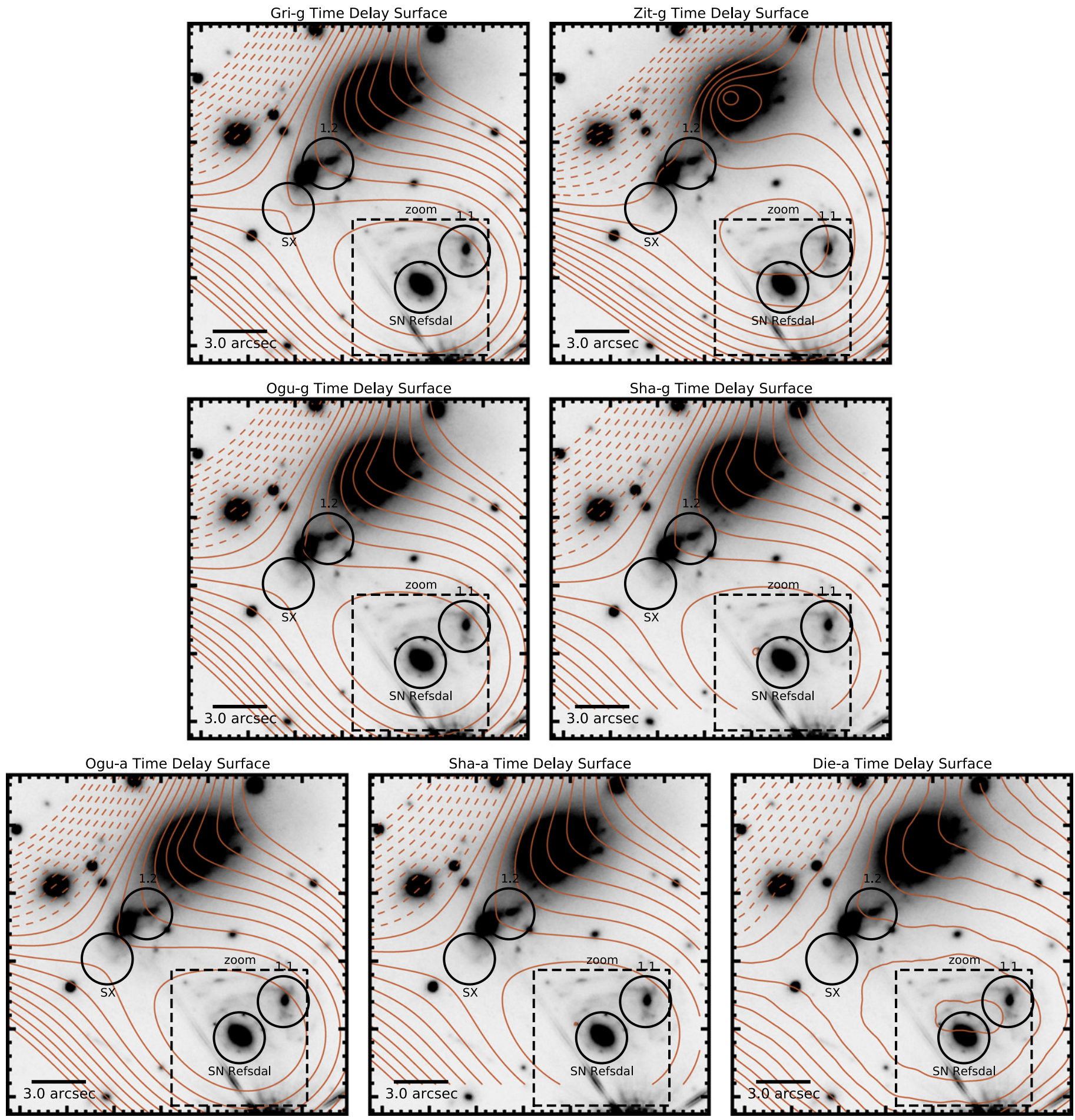

Figure 7. Time-delay surface details in the region marked in Figure 6. The dashed boxes mark the location of the zoom-in regions shown in Figure 8. Contour levels indicate the time delay from -5 to 5 years in increments of 0.5 years, relative to S1. Negative levels are marked by dashed contours. The gray-scale background image shows the HFF F140W epoch2 version 1.0 mosaic.

Ogu-a ones. This is expected, considering that they have less flexibility than the free-form model. What is surprising, however, is that they also obtain the smaller rms residual scatter in the predicted versus observed image positions (Table 5). The Zit-g LTM model is perhaps the least flexible, in the sense that it cannot account for systematic variations in the projected $M / L$ ratio. This appears to be reflected in its overall largest rms residual scatter. When comparing the Die-a to the Zit-g model, we note that the former uses significantly more constraints than the latter. This may explain why, even though Die-a is in principle more flexible, it ends up estimating generally smaller uncertainties than Zit-g. 

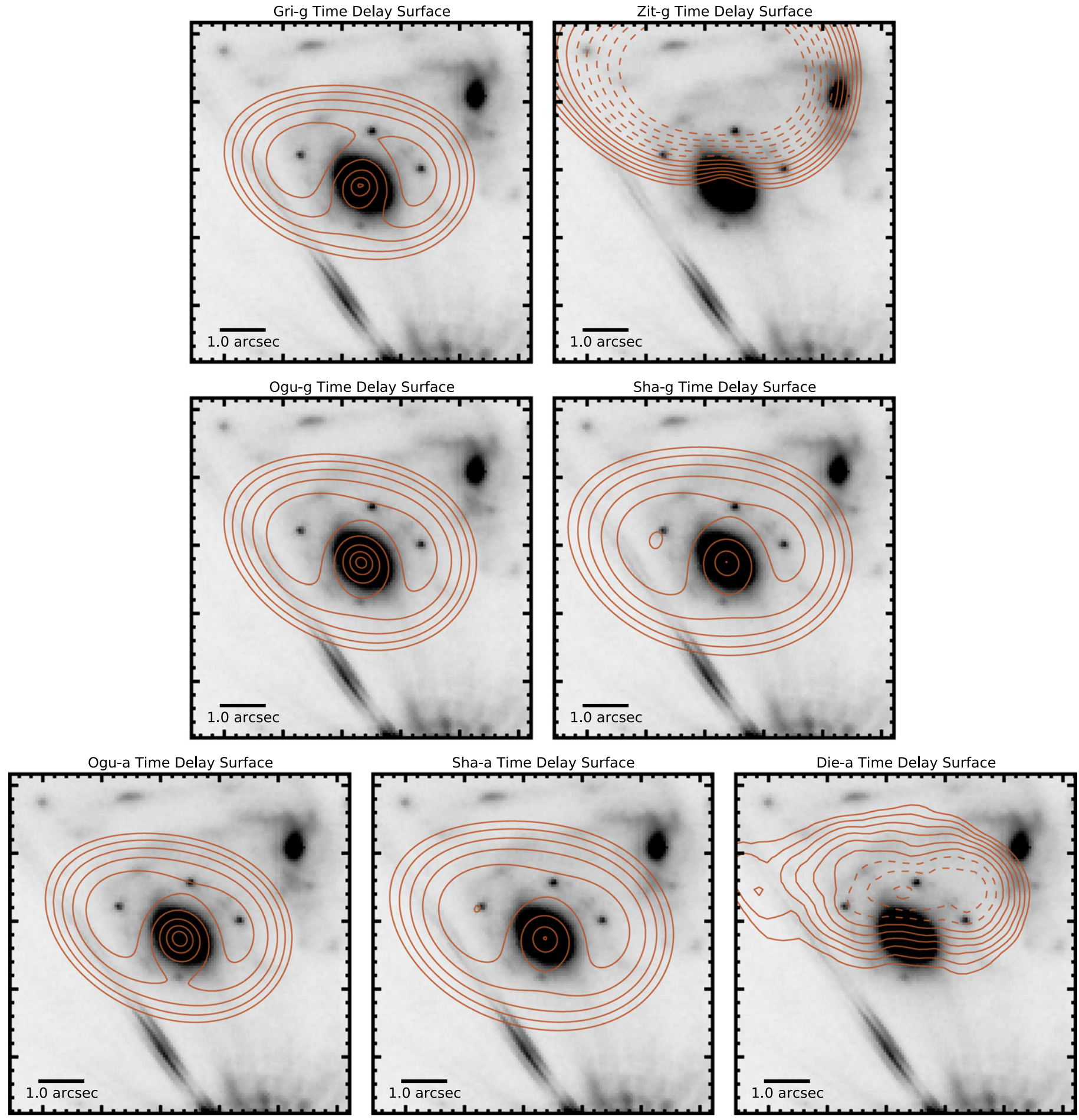

Figure 8. Time-delay surface details in the region marked in Figure 7. Contour levels indicate the time delay from -50 to 50 days in increments of 10 days, relative to S1. Negative levels are marked by dashed contours. The gray-scale background image shows the HFF F140W epoch2 version 1.0 mosaic.

\subsection{Forecasts for SN Refsdal: Peak Appearance and Brightness}

Figure 11 compares the prediction for the next appearance of SN Refsdal, near image 1.2 of the spiral galaxy (hereafter SX/ 1.2). All the models considered here predict the image to peak between the end of 2015 and the first half of 2016. We note that S1 was first discovered six months before its peak with F160W AB magnitude 25.5 (Kelly et al. 2015), and it peaked at $\mathrm{F} 160 \mathrm{~W} \approx 24.5 \mathrm{AB}$ (P. L. Kelly et al. 2015, in preparation;
Rodney et al. 2015, in preparation). Image SX/1.2 is predicted to be approximately $1 / 3$ as bright as image S1 (Figure 12), so it should be $\sim 26.7 \mathrm{mag}$ six months before peak and $\sim 25.7 \mathrm{mag}$ at peak. No image is detected in the vicinity of SY/1.3 in data taken with HST up until MACSJ1149.5+2223 became unobservable at the end of July, allowing us to rule out predicted peak times until 2016 January.

Remarkably, the models are in excellent mutual agreement regarding the next appearance of SN Refsdal. All of the predictions agree on the first trimester of 2016 as the most 


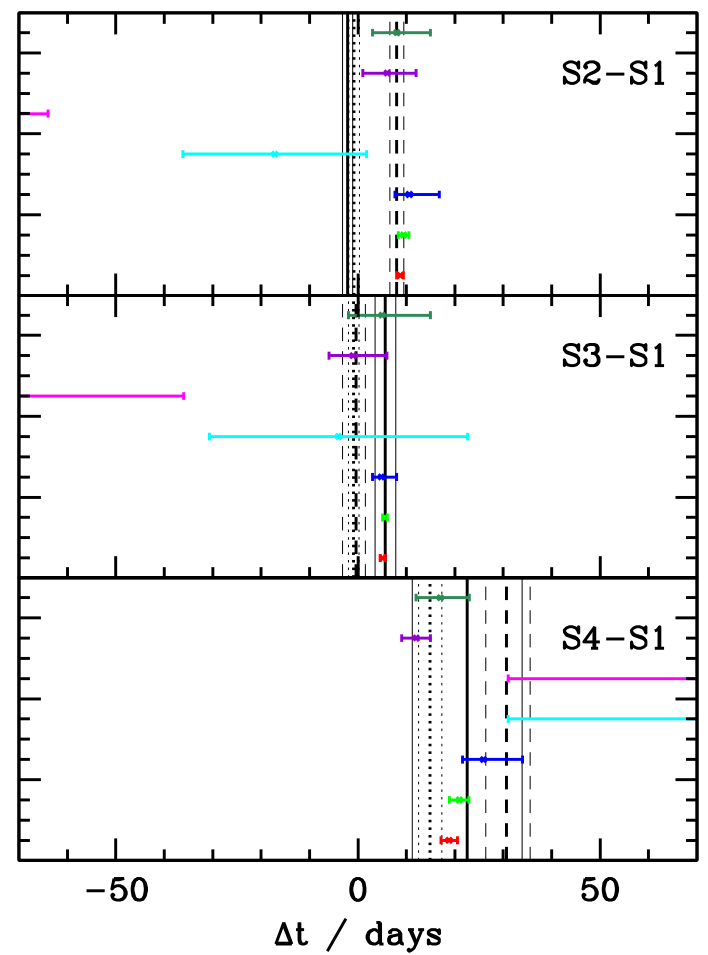

Sha-a

Sha $-\mathrm{g}$

Zit $-\mathrm{g}$

Die-a

Gri-g

Ogu-a

Ogu $-\mathrm{g}$

\section{$\Delta \mathrm{t} /$ days}

Figure 9. Observed (solid vertical line represents the preliminary measurements; dashed vertical line represents the updated template-based measurements; dotted vertical line represents the updated polynomial-based measurements; thin lines represent the $68 \%$ confidence range for each measurement) and predicted (points with error bars) time delays for the images in the cross configuration, relative to S1. Uncertainties represent the $68 \%$ confidence interval.

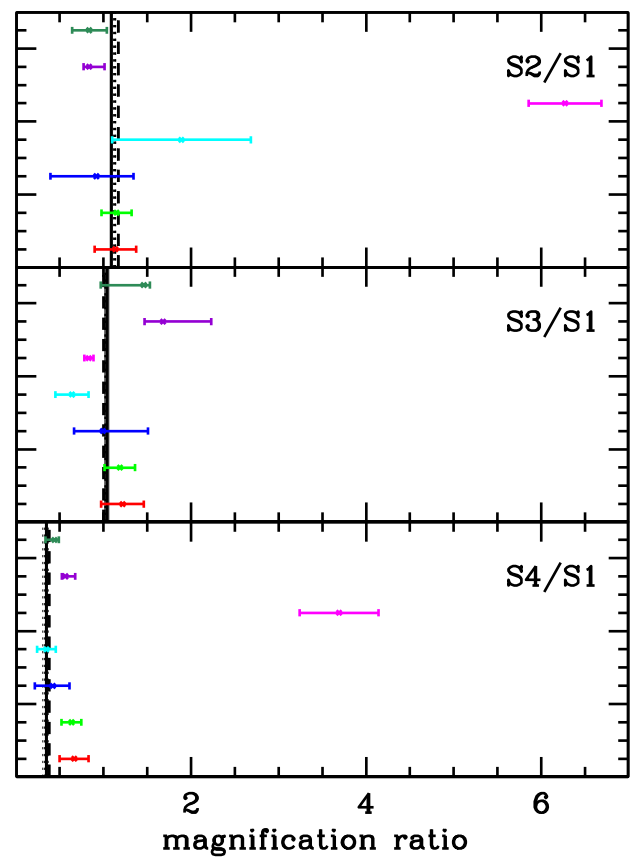

Sha-a

Sha $-\mathrm{g}$

Zit-g

Die-a

Gri-g

Ogu $-\mathrm{a}$

Ogu-g

Figure 10. Observed (lines as in Figure 9) and predicted (points with error bars) magnification ratios (absolute values) for the images in the cross configuration, relative to $\mathrm{S} 1$.

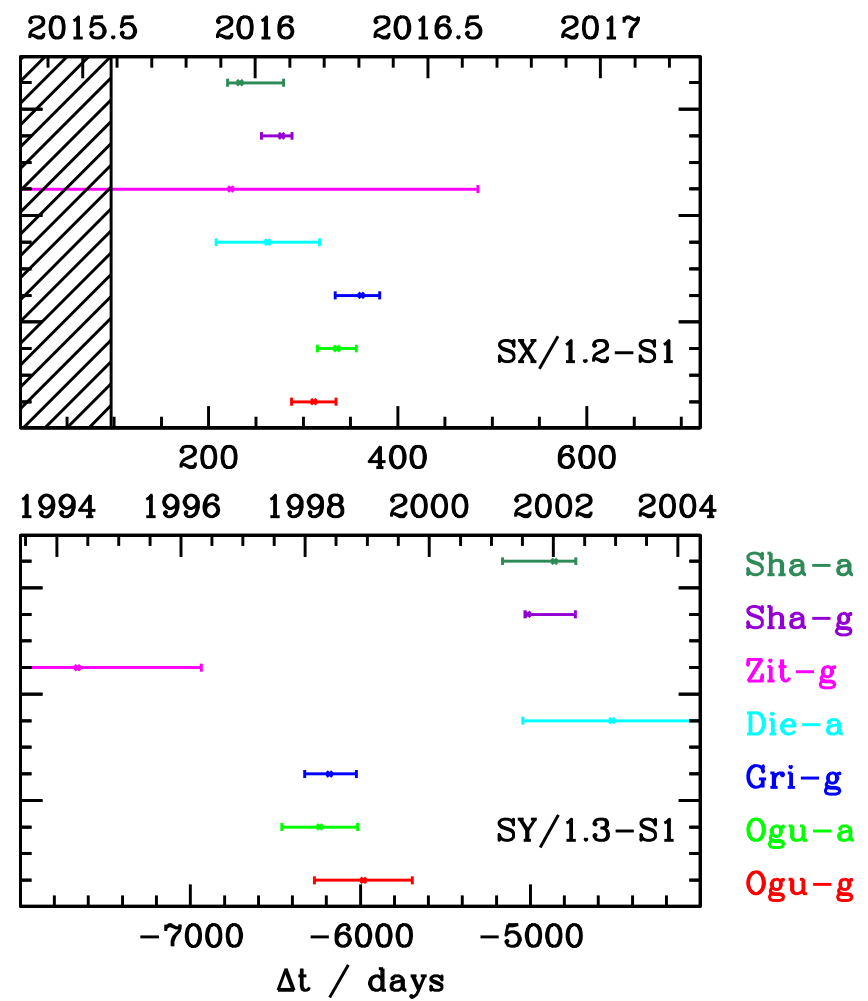

Figure 11. Predicted time delays for the more distant images, relative to S1. The top scale gives the expected date of the peak brightness of the image, with an uncertainty of \pm 20 days given by the uncertainty in the date of the peak of the observed images (to preserve full blindness we adopt here the preliminary peak measurement 2015 April 26, and not the revised measurement 2015 April 20 ; they are consistent within the uncertainties of \pm 20 days). The hatched region is ruled out by past $H S T$ observations.
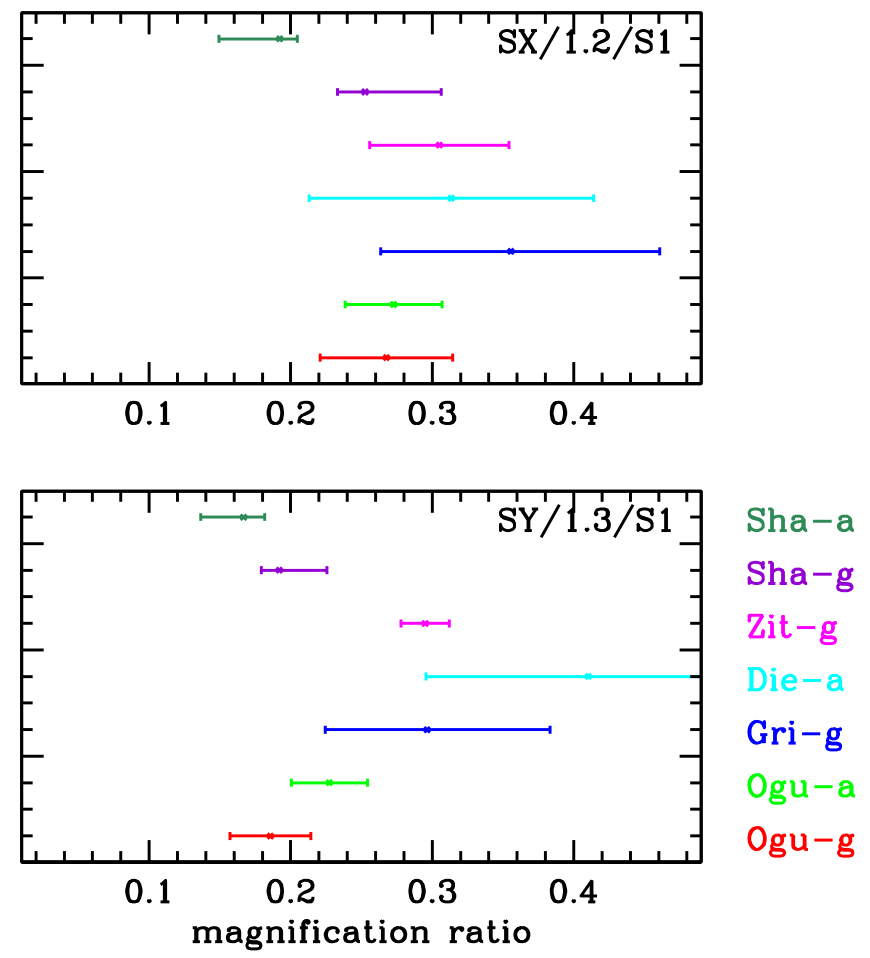

Figure 12. Predicted magnification ratios (absolute values) for the more distant images, relative to $\mathrm{S} 1$. 
likely date of the peak. Sha-a is the only one that predicts a slightly fainter flux with a magnification ratio $\left(0.19_{-0.04}^{+0.01}\right)$ as opposed to the $\sim 1 / 3$ value predicted by the other models. Interestingly, Zit-g has the largest uncertainty on time delay, but not on magnification ratio. As in the case of the cross configuration, the "simply parametrized" models yield the smallest uncertainties.

Unfortunately, the model-based estimates of the past appearance of SN Refsdal cannot be tested by observations. The image near 1.3 (hereafter SY/1.3) is estimated to have been significantly fainter than $\mathrm{S} 1$, and thus undetectable from the ground, at a time when WFC3-IR was not available. The images of MACSJ1149.5+2223 taken in the optical with ACS in 2004 April (GO-9722, PI Ebeling; $3 \sigma$ limit F814W $\mathrm{AB}=27.0 \mathrm{mag}$ ) are not sufficiently deep to set any significant constraints, considering the peak brightness of S1 in F814W was $\sim 27 \mathrm{mag}$, and we expect SY $/ 1.3$ to be $0.75-2$ mag fainter. As a purely theoretical exercise it is interesting to notice that the time delay varies dramatically between models, differing by almost 10 years between the Zit-g and the Sha-a, Sha-g, and Die-a models. Remarkably, and similarly to what was seen for the cross configuration, the magnifications are in significantly better agreement.

\section{DISCUSSION}

In this section we briefly discuss our results, first by recapitulating the limitations of our analysis (Section 6.1), and then by comparing them with previous work (Section 6.2).

\subsection{Limitation of the Blind Test and of the Models}

SN Refsdal gives us a unique opportunity to test our models blindly. However, in order to draw the appropriate conclusions from this test, we need to be aware of the limitations of both the test and the models.

The first limitation to keep in mind is that this test is very specific. We are effectively testing point-like predictions of the lensing potential and its derivatives. Similarly to the case of SN "Tomas" (Rodney et al. 2015), it is very hard to generalize the results of this test even to the strong lensing area shown in our maps. More global metrics should be used to infer a more global assessment of the quality of the models. An example of such a metric is the rms scatter between the image positions given in Table 5, even though of course even this metric does not capture all of the features of a model. For example, the rms does not capture how well the model reproduces time delays and magnifications, in addition to positions, and one could imagine trading one for the other.

It is also important to remind ourselves that whereas the magnification and time delays at specific points may vary significantly between models, other quantities that are more relevant for statistical use of clusters as cosmic telescopes, such as the area in the source plane, are much more stable (e.g., Wang et al. 2015). And, of course, other quantities such as colors and line ratios are not affected at all by gravitational lensing. It would be interesting to find ways to carry out true observational tests of more global predictions of lens models. One way to achieve this would be to carry out tests similar to those afforded by SN Tomas and SN Refsdal on a large sample of clusters. Another possibility could be to reach sufficiently deep that the statistical properties of the background sources (e.g., the luminosity function) are measured with sufficient
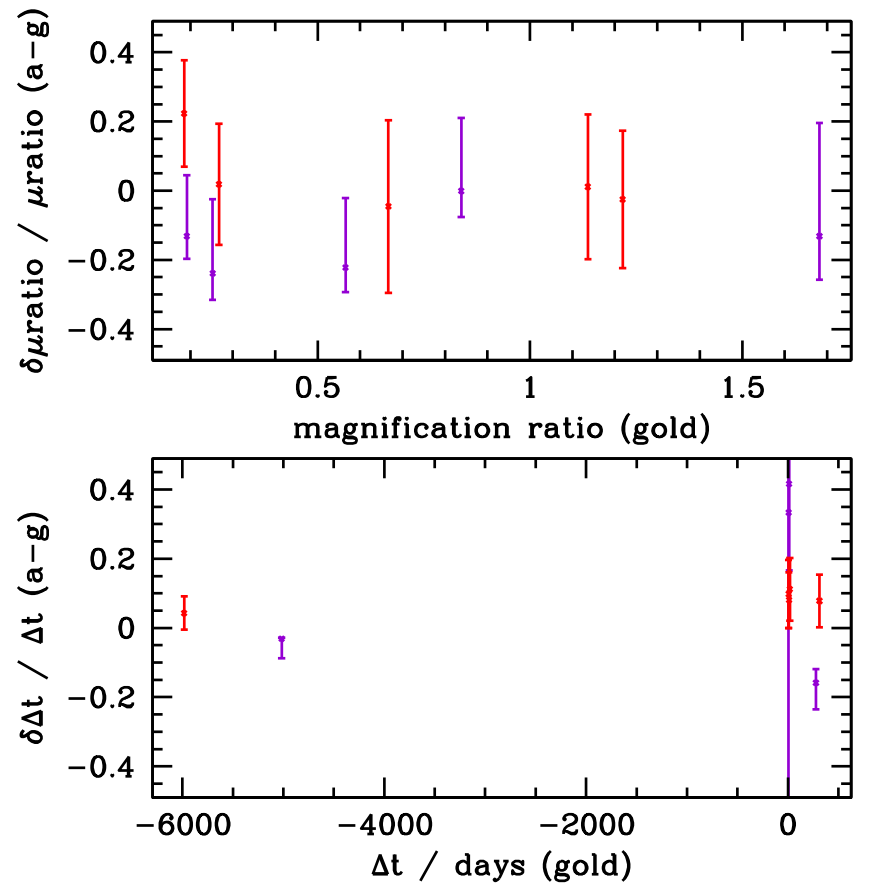

Figure 13. Relative change in predicted time delays (bottom) and magnification ratio (top) between "a" and "g" models. Dark purple points represent the models by Sharon et al., while red points represent the models by Oguri et al. Vertical bars represent the statistical uncertainties on the "g" models.

precision and small enough cosmic variance to allow for meaningful tests of model uncertainties. Alternatively, tests against simulated data are certainly informative (e.g., M. Meneghetti et al. 2015, in preparation), although their results should also be interpreted with great care, as they depend crucially on the fidelity of the simulated data and the cross-talk between methods used to simulate the data and those used to carry out the inference.

The second limitation to keep in mind is that the uncertainties listed in this paper are purely statistical in nature. As for the case of image positions-where the rms scatter is typically larger than the astrometric precision of the image positions themselves (consistent with the fact that there are residual systematics in cluster lens modeling owing to known effects such as substructure, eg, Bradač et al. 2009)—we should not expect the time delays and magnifications to be perfectly reproduced by the models either. The spread between the different model predictions gives us an idea of the so-called model uncertainties, even though unfortunately they cannot be considered an exact measurement. The spread could be exaggerated by inappropriate assumptions in some of the models, or underestimated if common assumptions are unjustified.

We can use the fact that Oguri et al. and Sharon et al. each submitted two models to estimate the uncertainty relative to the choice of multiple images. By comparing the predictions of the Sha-a and Sha-g and of the Ogu-a and Ogu-g models, we can measure how much the predictions of the models change by adding nonspectroscopically confirmed images to the gold sample, keeping everything else fixed. As shown in Figure 13, the predicted magnification ratios change by less than the statistical uncertainties. The changes in predicted time delays are slightly larger, comparable to the estimated statistical 
uncertainties, although the relative change in time delays is perhaps not the best metric for the short time delays in the Einstein cross configuration. As can be seen in Figures 9 and 11 , the absolute change in predicted time delays is typically within the estimated statistical uncertainties. From this test we conclude that in this case deciding whether to consider a secure but not spectroscopically confirmed set of multiple images introduces an uncertainty that is subdominant with respect to the statistical uncertainties. This is consistent with our expectation that the enlarged set of multiple images does not contain false candidates. In interpreting this result, however, we have to keep in mind the locality of this test and the fact that the nearest images to the observed and predicted images of SN Refsdal are the knots of its host galaxy, all at the same known spectroscopic redshift. Thus, it would have been surprising to find a large difference at these locations.

As already mentioned in the introduction, other potential sources of uncertainty are related to the mass-sheet degeneracy and its generalizations (Falco et al. 1985; Schneider \& Sluse 2013, 2014), the effects of structure along the line of sight (Dalal et al. 2005), and multiplane lensing (McCully et al. 2014; Schneider 2014). All of the models considered here are single-plane lens models. They break the mass-sheet degeneracy by assuming that the surface mass density profile goes to zero at infinity with a specific radial dependency.

On the scale of the known images of SN Refsdal, the measured time delays and magnification ratios give us a way to estimate these residual uncertainties. The reasonably good agreement between the model prediction and measurements shows that these (systematic) "unknown unknowns" are not dominant with the respect to the (statistical) "known unknowns." However, since the agreement is not perfect, we conclude that the "unknown unknowns" are not negligible either. We can perhaps use the experience gathered in the study of time delays of lensed quasars to estimate the amplitude of the line-of-sight uncertainties. On scales similar to that of the known images of SN Refsdal, they are believed to be up to $\sim 10 \%$ before corrections for galaxies not in clusters (Suyu et al. 2010; Collett et al. 2013; Greene et al. 2013; Suyu et al. 2014). In numerical simulations, the line-of-sight effects appear to increase with the measured overdensity of galaxies (Greene et al. 2013), so it is possible that they are larger for an overdense region like that of MACSJ1149.5+2223.

On galaxy scales, breaking the mass-sheet degeneracy using stellar kinematics and physically motivated galaxy models appears to produce results consistent with residual uncertainties on the order of a few percent (Suyu et al. 2014). On cluster scales, the degeneracy is partly broken by the use of multiple images at different redshifts (e.g., Bradač et al. 2004a). However, in the absence of nonlensing data, we cannot rule out that the residual mass-sheet degeneracy is the dominant source of systematic uncertainty. Assessing the uncertainties related to multiplane effects would require knowledge of the mass distribution in three dimensions and is beyond the scope of the present work. Thus, multiplane lensing cannot be ruled out as a significant source of systematic uncertainty for the prediction of the time delay and magnification ratios of the known images of SN Refsdal. As far as the future image of SN Refsdal is concerned, future observations will tell us how much our uncertainties are underestimated owing to unknown systematics.
Finally, we remind the reader that although for this analysis we kept fixed the cosmological parameters, they are a (subdominant) source of uncertainty. To first order, the timedelay distance is proportional to the Hubble constant, so there is at least a $3 \%$ systematic uncertainty (Riess et al. 2011; Freedman et al. 2012) on our predicted time delays (and typically $5 \%-10 \%$ when considering all of the other parameters, depending on assumptions and priors; Suyu et al. 2013, 2014).

\subsection{Comparison with Previous Models}

We can get a quantitative sense of the improvement of the mass models as a result of the new data by comparing how the prediction of the time delay and magnification ratios have changed for the teams who had previously published predictions.

\subsubsection{Previous Models by Members of our Team}

The Zit-g model updates the models developed by A. Z. for the SN Refsdal discovery paper (Kelly et al. 2015). The Zit-g model supersedes the estimates of time delays and magnifications given in the original paper by providing predictions as well as quantitative uncertainties.

The update of the Oguri (2015) model presented here changes the time delays for S2, S3, S4, SX, SY from 9.2, 5.2, $22.5,357,-6193$ days to $8.7 \pm 0.7,5.1 \pm 0.5,18.8 \pm 1.7$, $311 \pm 23.6,-5982 \pm 287$ days, respectively (for the Ogu-g model, see plot for Ogu-a). Thus, the predicted time delays have changed by less than $1-2 \sigma$, with the inclusion of additional data. The magnification ratios have been similarly stable. The main effect of the additional data has been to reduce the uncertainties.

The update of the Sharon \& Johnson (2015) model presented here changes the time delays for S2, S3, S4, SX, SY from $2_{-6}^{+10}$, $-5_{-7}^{+13}, 7_{-3}^{+16}, 237_{-50}^{+37}+4251_{-373}^{+369}$ days to $8_{-5}^{+7}, 5_{-7}^{+10}, 17_{-5}^{+6}$, $233_{-13}^{+46},-4860_{-305}^{+126}$ days, respectively (for the Sha-a model, see Table 6 for Sha-g). Thus, the predicted time delays have changed by less than $1-2 \sigma$, with the inclusion of additional data, especially the new spectroscopic redshifts (the list of multiple images is very similar). The magnification ratios have been similarly stable. The main effect of the additional data has been to reduce the uncertainties.

Diego et al. (2015) do not give time delays for the cross configuration, owing to the limitations inherent to keeping the $M / L$ of the galaxy in the middle of the cross fixed to the global value. Their predictions for the long delays SX and SY have changed with the inclusion of new data from $375 \pm 25$ to $262 \pm 54$ days, and from $-3325 \pm 762$ to $-4521 \pm 524$. Interestingly, the uncertainties in the future delay have increased with the new data, which may be caused by the correction of previously erroneous inputs, like the redshift of system 3, and also to the increased range of models and grid parameters considered here. The fact that the predictions changed by more than the estimated uncertainties is consistent with our previous conclusion that the statistical uncertainties underestimate the total uncertainty.

\subsubsection{Jauzac et al.}

During the final stages of the preparation of this manuscript, Jauzac et al. (2015) posted on the arxiv another independent model of MACSJ1149.5+2223. Their model is based on a 
subset of the data presented here, and different sets of multiple images and knots in the spiral host galaxy. Comparing only the systems with spectroscopic redshifts, our analyses agree on multiply imaged systems 1, 2, 3, 4, 5, 110 (22 in their nomenclature), and in rejecting the identification of system 12 as multiply imaged. We do not use system 9, for which they obtain a spectroscopic redshift of 0.981 . We obtain spectroscopic redshifts for systems 13 and 14 (1.24 and 3.70), which are statistically somewhat in tension with their model redshifts of $1.28 \pm 0.01$ and $3.50 \pm 0.06$. Their catalog comprises 57 spectroscopically confirmed cluster members, while ours consists of 170. Thus, the Jauzac et al. (2015) model is not directly comparable to the models presented here. However, it provides a useful additional comparison for this forecast. We note that Jauzac et al. (2015) include the main developers of Lenstool, the "simply parametrized" lens modeling software used by Sharon et al. for the analysis presented in this paper. The difference in the predictions between the two teams highlights how systematic differences can arise from input data and modelers' choices, as well as from assumptions of each modeling method.

The Jauzac et al. (2015) model $^{33}$ predicts time delays and magnification ratios that are significantly different from the ones actually observed from the cross configuration. When comparing their prediction with observations, all of the limitations discussed in Section 6.1 should be kept in mind, as they apply to the Jauzac et al. models as well. Furthermore, as Jauzac et al. (2015) point out, their predictions for the cross configuration are very sensitive to the mass density profile assumed for the cluster galaxy closest to it. Therefore, the random uncertainties underestimate the total uncertainties.

Their predicted time delays for S2-S1, S3-S1, and S4-S1 are $90 \pm 17,30 \pm 35$, and $-60 \pm 41$ days, respectively, to be compared with the measured values given in Table 1. Considering their statistical uncertainties, which are much larger than the measurement uncertainties, the time delays are within $\sim 5 \sigma, 1 \sigma$, and $2 \sigma$ of the measurements. The disagreement with the S2-S1 time delay is especially remarkable considering that all the other models predict the two images to be almost simultaneous. The flux ratios $(0.86 \pm 0.13,0.89 \pm 0.11$, and $0.42 \pm 0.05$ for $\mathrm{S} 2 / \mathrm{S} 1, \mathrm{~S} 3 / \mathrm{S} 1, \mathrm{~S} 4 / \mathrm{S} 1$, respectively) are also somewhat in tension with the measured values, although the disagreement is in line with that of the models presented in this paper. It would be interesting to update the Jauzac et al. (2015) model, correcting the redshifts of systems 13 and 14 to see if this could reduce the discrepancy. Overall, it is interesting to note that Jauzac et al. (2015) predict the magnifications with higher accuracy than the time delays, even though magnifications are potentially more sensitive to local substructure (millilensing) and microlensing effects.

The time delay predicted by Jauzac et al. (2015) for image $\mathrm{SX} / 1.2$ is significantly longer than for the models presented here, pushing the next appearance of the peak to the middle of 2016. The time delay of image SY/1.3 is shorter than for most models presented here, but unfortunately not short enough to be testable with archival observations. Incidentally, Jauzac et al. (2015) also predict $S Y / 1.3$ to be fainter than in the models presented here $(0.16 \pm 0.02$ of the brightness of $\mathrm{S} 1)$,

\footnotetext{
33 We refer here to version 3 of the Jauzac et al. (2015) paper, which appeared on the arxiv on 2015 October 13. The predictions have changed significantly between versions 2 (2015 October 1) and 3, owing to the improved treatment of the cluster galaxy nearest to the cross configuration.
}

which makes this prediction even more difficult to test with archival data.

\section{SUMMARY}

SN Refsdal gives us a unique opportunity to carry out a truly blind test of cluster-scale gravitational lens models. In order to make the most of this opportunity, we have used an unprecedented combination of imaging and spectroscopic data as input for 7 lens models, based on 5 independent techniques. The models have been tested against independent measurements of time delays and magnification ratios for the known images of SN Refsdal and used to predict its future (and past) appearance. Our main results can be summarized as follows.

1. We have collected 429 spectroscopic redshifts in the field of MACSJ1149.5+2223 from VLT-MUSE (Grillo et al. 2015a), Keck DEIMOS, and HST-WFC3 (Schmidt et al. 2014; G. B. Brammer et al. 2015, in preparation; Treu et al. 2015) observations. These include 170 spectroscopic cluster members and 23 multiple images of 10 different galaxies.

2. We have collected measurements of time delays and magnification ratios for the known images of SN Refsdal (Rodney et al. 2015, in preparation).

3. We have compiled and expanded a list of candidate multiply imaged galaxies and multiply imaged knots in the host galaxy of SN Refsdal. All images have been vetted by a group of expert classifiers, resulting in a list of gold and silver-quality images.

4. The seven lens models have remarkably good fidelity, with residual rms scatter between observed and predicted image positions ranging between 0 ." 16 and 1 ."3.

5. The model predictions agree reasonably well with the observed delays and magnifications of SN Refsdal (within 68\%-95\% uncertainty, or 10 days in the case of S2-S1), showing that unknown systematics are comparable to or smaller than the calculated statistical uncertainties.

6. All models predict that an image of SN Refsdal will appear near the SX/1.2 location between the submission of this paper and the beginning of 2016. The most likely time for the peak is the first trimester of 2016. Given the slow rise of the light curve of SN Refsdal and the predicted brightness of $\mathrm{SX} / 1.2$, the image could appear as soon as MACSJ1149.5+2223 is visible again by HSTWFC3 at the end of 2015 October.

7. The past appearance of SN Refsdal near position SY/1.3 would have been too faint to be detectable in existing archival images, and thus cannot be tested.

There are two possible outcomes to the work presented in this paper. First, our predictions could be proven correct. This outcome would be an encouraging sign that all the efforts by the community to gather data and improve lens modeling tools are paying off. If, alternatively, our predictions turn out to be wrong, we will have to go back to the drawing board, having learned an important lesson about systematic uncertainties.

Note Added in Proof. After the acceptance of this manuscript, the predicted image SX was discovered in HST images taken in November and December 2015. The discovery and comparison with our model predictions is predicted by Kelly et al. (2016). Also after the acceptance of this manuscript, it was discovered 
that the predictions of the Zit-g lens model are inaccurate, due to numerical resolution insufficient to correctly resolve the vicinity of the Einstein Cross configuration S1-S4. The quantities affected are the panels labeled Zit-g in Figures 48; the points labeled Zit-g in Figures 9-12; and the last line of Table 6. While calculations at higher resolution yield qualitatively the same results, nevertheless they have smaller uncertainties and are in better agreement with the observed S1$\mathrm{S} 4$ time delays and magnification ratio.

The numerical inaccuracy of the Zit-g model does not affect in any way the other results in the paper, including redshifts, arcs and knots identifications, measured time delays, and the predictions of the six other lens models. Furthermore, since the qualitative description of the Zit-g model and its predictions are correct, the discussion and conclusions of the paper are not affected in any way.

The error was discovered after the blind test deadline ( 30 October 2015, date of the first HST observations of the field). Thus, in order to preserve the blindness of the models described in this paper, the corrected results will be presented in a future publication.

The authors thank Raphael Gavazzi, Mathilde Jauzac, and Jens Hjorth for insightful comments on the manuscript. This work is supported by NASA through grants HST-GO-13459 and HST-GO-14041 from the Space Telescope Science Institute (STScI), which is operated by the Association of Universities for Research in Astronomy, Inc., under NASA contract NAS 5-26555. T.T. gratefully acknowledges the hospitality of the American Academy in Rome and of the Observatorio di Monteporzio Catone, where parts of this manuscript were written. T.T. is supported by the Packard Foundation in the form of a Packard Research Fellowship. J.M.D. is grateful for support from the consolider project CSD2010-00064 and AYA2012-39475-C02-01 funded by the Ministerio de Economia y Competitividad. A.V.F.'s research is supported by the Christopher R. Redlich Fund, the TABASGO Foundation, and NSF grant AST-1211916. C.G. acknowledges support by the VILLUM FONDEN Young Investigator Programme through grant no. 10123. Support for A.Z. was provided by NASA through Hubble Fellowship grant \#HSTHF2-51334.001-A awarded by STScI. The work of M.O. was supported in part by World Premier International Research Center Initiative (WPI Initiative), MEXT, Japan, and Grant-inAid for Scientific Research from the JSPS (26800093). Financial support for this work was provided to S.A.R. by NASA through grants HST-HF-51312 and HST-GO-13386 from STScI. A.H. is supported by NASA Headquarters under the NASA Earth and Space Science Fellowship Program, grant ASTRO14F-0007. R.J.F. gratefully acknowledges support from NSF grant AST-1518052, NASA grants HST-GO-14041 and HST-GO-13386, and the Alfred P. Sloan Foundation. This work was supported in part by a NASA Keck PI Data Award (PID 47/2014B_N125D, PI Jha).

We are very grateful to the staff of the Hubble Space Telescope for their assistance in planning, scheduling, and executing the HST observations used in this work. We thank the STScI and ESO directors for granting Directory Discretionary time to allow for timely follow-up observations of SN Refsdal. Some of the data presented herein were obtained at the W. M. Keck Observatory from telescope time allocated to NASA through the agency's scientific partnership with the
California Institute of Technology and the University of California; the Observatory was made possible by the generous financial support of the W. M. Keck Foundation. The authors wish to recognize and acknowledge the very significant cultural role and reverence that the summit of Mauna Kea has always had within the indigenous Hawaiian community. We are most fortunate to have the opportunity to conduct observations from this mountain.

\section{REFERENCES}

Bertin, E., \& Arnouts, S. 1996, A\&AS, 117, 393

Blandford, R., \& Narayan, R. 1986, ApJ, 310, 568

Bolton, A. S., \& Burles, S. 2003, ApJ, 592, 17

Bradač, M., Lombardi, M., \& Schneider, P. 2004a, A\&A, 424, 13

Bradač, M., Schneider, P., Lombardi, M., et al. 2004b, A\&A, 423, 797

Bradač, M., Treu, T., Applegate, D., et al. 2009, ApJ, 706, 1201

Brammer, G. B., van Dokkum, P. G., Franx, M., et al. 2012, ApJS, 200, 13 Broadhurst, T., Benítez, N., Coe, D., et al. 2005, ApJ, 621, 53

Coe, D., Bradley, L., \& Zitrin, A. 2015, ApJ, 800, 84

Collett, T. E., Marshall, P. J., Auger, M. W., et al. 2013, MNRAS, 432, 679

Dalal, N., Hennawi, J. F., \& Bode, P. 2005, ApJ, 622, 99

Diego, J. M., Broadhurst, T., Chen, C., et al. 2016, MNRAS, 456, 356

Diego, J. M., Protopapas, P., Sandvik, H. B., \& Tegmark, M. 2005, MNRAS, 360,477

Diego, J. M., Tegmark, M., Protopapas, P., \& Sandvik, H. B. 2007, MNRAS, 375,958

Dobler, G., \& Keeton, C. R. 2006, ApJ, 653, 1391

Ebeling, H., Barrett, E., Donovan, D., et al. 2007, ApJL, 661, L33

Elíasdóttir, Á, Limousin, M., Richard, J., et al. 2007, arXiv:0710.5636

Faber, S. M., Phillips, A. C., Kibrick, R. I., et al. 2003, Proc. SPIE, 4841, 1657

Falco, E. E., Gorenstein, M. V., \& Shapiro, I. I. 1985, ApJL, 289, L1

Freedman, W. L., Madore, B. F., Scowcroft, V., et al. 2012, ApJ, 758, 24

Goobar, A., Mörtsell, E., Amanullah, R., \& Nugent, P. 2002, A\&A, 393, 25

Greene, Z. S., Suyu, S. H., Treu, T., et al. 2013, ApJ, 768, 39

Grillo, C., Karman, W., Suyu, S. H., et al. 2015a, ApJ, submitted, (arXiv:1511.04093)

Grillo, C., Suyu, S. H., Rosati, P., et al. 2015b, ApJ, 800, 38

Holz, D. E. 2001, ApJL, 556, L71

Ishigaki, M., Kawamata, R., Ouchi, M., et al. 2015, ApJ, 799, 12

Jauzac, M., Richard, J., Limousin, M., et al. 2015, MNRAS, submitted (arXiv:1509.08914)

Johnson, T. L., Sharon, K., Bayliss, M. B., et al. 2014, ApJ, 797, 48

Jullo, E., \& Kneib, J.-P. 2009, MNRAS, 395, 1319

Jullo, E., Kneib, J.-P., Limousin, M., et al. 2007, NJPh, 9, 447

Karman, W., Caputi, K. I., Grillo, C., et al. 2015a, A\&A, 574, A11

Karman, W., Grillo, C., Balestra, I., et al. 2016, A\&A, 585, A27

Kassiola, A., \& Kovner, I. 1993, ApJ, 417, 450

Kawamata, R., Oguri, M., Ishigaki, M., Shimasaku, K., \& Ouchi, M. 2015, ApJ, submitted (arXiv:1510.06400)

Kelly, P. L., Rodney, S. A., Treu, T., et al. 2015, Sci, 347, 1123

Kelly, P. L., Rodney, S. A., Treu, T., et al. 2016, ApJL, submitted (arXiv:1512.04654)

Kolatt, T. S., \& Bartelmann, M. 1998, MNRAS, 296, 763

Limousin, M., Kneib, J.-P., \& Natarajan, P. 2005, MNRAS, 356, 309

Linder, E. V., Wagoner, R. V., \& Schneider, P. 1988, ApJ, 324, 786

McCully, C., Keeton, C. R., Wong, K. C., \& Zabludoff, A. I. 2014, MNRAS, 443, 3631

Meylan, G., Jetzer, P., North, P. et al. (ed.) 2006, Gravitational Lensing: Strong, Weak and Micro (Berlin: Springer)

Momcheva, I. G., Brammer, G. B., van Dokkum, P. G., et al. 2015, ApJS, submitted (arXiv:1510.02106)

Navarro, J. F., Frenk, C. S., \& White, S. D. M. 1997, ApJ, 490, 493

Nordin, J., Rubin, D., Richard, J., et al. 2014, MNRAS, 440, 2742

Oguri, M. 2010, PASJ, 62, 1017

Oguri, M. 2015, MNRAS, 449, L86

Oguri, M., Bayliss, M. B., Dahle, H., et al. 2012, MNRAS, 420, 3213

Oguri, M., \& Kawano, Y. 2003, MNRAS, 338, L25

Oguri, M., \& Marshall, P. J. 2010, MNRAS, 405, 2579

Oguri, M., Schrabback, T., Jullo, E., et al. 2013, MNRAS, 429, 482

Oguri, M., Suto, Y., \& Turner, E. L. 2003, ApJ, 583, 584

Oke, J. B., \& Gunn, J. E. 1983, ApJ, 266, 713

Patel, B., McCully, C., Jha, S. W., et al. 2014, ApJ, 786, 9 
Popper, K. R. 2002, The logic of scientific discovery (Translated from German original: Logik der Forschung. Zur Erkenntnistheorie der Modernen Naturwissenschaft) (London: Routledge)

Postman, M., Coe, D., Benítez, N., et al. 2012, ApJS, 199, 25

Quimby, R. M., Oguri, M., More, A., et al. 2014, Sci, 344, 396

Refsdal, S. 1964, MNRAS, 128, 307

Richard, J., Jauzac, M., Limousin, M., et al. 2014, MNRAS, 444, 268

Riess, A. G., Macri, L., Casertano, S., et al. 2011, ApJ, 730, 119

Rodney, S. A., Patel, B., Scolnic, D., et al. 2015, ApJ, 811, 70

Rodney, S. R., Strolger, L. G., Kelly, P. L., et al. 2016, ApJ, submitted (arXiv:1512.05734)

Schmidt, K. B., Treu, T., Brammer, G. B., et al. 2014, ApJL, 782, L36

Schneider, P. 1985, A\&A, 143, 413

Schneider, P. 2014, A\&A, 568, L2

Schneider, P., \& Sluse, D. 2013, A\&A, 559, A37

Schneider, P., \& Sluse, D. 2014, A\&A, 564, A103

Sendra, I., Diego, J. M., Broadhurst, T., \& Lazkoz, R. 2014, MNRAS, 437,2642

Shapiro, I. I. 1964, PhRvL, 13, 789

Sharon, K., \& Johnson, T. L. 2015, ApJL, 800, L26

Smith, G. P., Ebeling, H., Limousin, M., et al. 2009, ApJL, 707, L163
Sullivan, M., Ellis, R., Nugent, P., Smail, I., \& Madau, P. 2000, MNRAS, 319,549

Suyu, S. H., Auger, M. W., Hilbert, S., et al. 2013, ApJ, 766, 70

Suyu, S. H., \& Halkola, A. 2010, A\&A, 524, A94

Suyu, S. H., Hensel, S. W., McKean, J. P., et al. 2012, ApJ, 750, 10

Suyu, S. H., Marshall, P. J., Auger, M. W., et al. 2010, ApJ, 711, 201

Suyu, S. H., Treu, T., Hilbert, S., et al. 2014, ApJL, 788, L35

Tewes, M., Courbin, F., \& Meylan, G. 2013a, A\&A, 553, A120

Tewes, M., Courbin, F., Meylan, G., et al. 2013b, A\&A, 556, A22

Treu, T. 2010, ARA\&A, 48, 87

Treu, T., \& Ellis, R. S. 2015, ConPh, 56, 17

Treu, T., Schmidt, K. B., Brammer, G. B., et al. 2015, ApJ, 812, 114

Wang, X., Hoag, A., Huang, K.-H., et al. 2015, ApJ, 811, 29

Xu, D., Sluse, D., Schneider, P., et al. 2016, MNRAS, 456, 739

Zheng, W., Postman, M., Zitrin, A., et al. 2012, Natur, 489, 406

Zitrin, A., \& Broadhurst, T. 2009, ApJL, 703, L132

Zitrin, A., Broadhurst, T., Bartelmann, M., et al. 2012a, MNRAS, 423, 2308

Zitrin, A., Broadhurst, T., Umetsu, K., et al. 2009, MNRAS, 396, 1985

Zitrin, A., Fabris, A., Merten, J., et al. 2015, ApJ, 801, 44

Zitrin, A., Meneghetti, M., Umetsu, K., et al. 2013, ApJL, 762, L30

Zitrin, A., Rosati, P., Nonino, M., et al. 2012b, ApJ, 749, 97 